\title{
First quantized approaches to neutrino oscillations and second quantization
}

\author{
C. C. Nishi* \\ Instituto de Física Teórica, UNESP-São Paulo State University, Rua Pamplona, 145, 01405-900-São Paulo, Brazil
}

(Received 13 September 2005; published 30 March 2006)

\begin{abstract}
Neutrino oscillations are treated from the point of view of relativistic first quantized theories and compared to second quantized treatments. Within first quantized theories, general oscillation probabilities can be found for Dirac fermions and charged spin 0 bosons. A clear modification in the oscillation formulas can be obtained and its origin is elucidated and confirmed to be inevitable from completeness and causality requirements. The left-handed nature of created and detected neutrinos can also be implemented in the first quantized Dirac theory in the presence of mixing; the probability loss due to the changing of initially left-handed neutrinos to the undetected right-handed neutrinos can be obtained in analytic form. Concerning second quantized approaches, it is shown in a calculation using virtual neutrino propagation that both neutrinos and antineutrinos may also contribute as intermediate particles. The sign of the contributing neutrino energy may have to be chosen explicitly without being automatic in the formalism. At last, a simple second quantized description of the flavor oscillation phenomenon is devised. In this description there is no interference terms between positive and negative components, but it still gives simple normalized oscillation probabilities. A new effect appearing in this context is an inevitable but tiny violation of the initial flavor of neutrinos. The probability loss due to the conversion of lefthanded neutrinos to right-handed neutrinos is also presented.
\end{abstract}

DOI: 10.1103/PhysRevD.73.053013

PACS numbers: 14.60.Pq, 03.65.Pm, 03.70.+k

\section{INTRODUCTION}

Compelling experimental evidence [1] has shown that neutrinos undergo flavor oscillations in vacuum. Consequently, this fact requires massive neutrinos with mixing. These ingredients are not present in the standard model of elementary particles. For this reason, on the one hand, neutrino oscillations can provide a direct window to probe physics beyond the standard model [2]. On the other hand, some theoretical studies of mixing in the context of quantum field theory (QFT) by Blasone and Vitiello (BV) $[3,4]$ show the mixing problem may be related to more fundamental issues such as unitarily inequivalent representations and the vacuum structure, and its study is theoretically interesting for its own sake.

Nevertheless, the simpler plane-wave quantum mechanical descriptions [5,6] seemed to be in accordance, in certain realistic limits, with more refined descriptions, including various ingredients, such as localization aspects [7-9], flavor current densities [10], influence of creation and detection processes [11,12], time-dependent perturbation theory [13], and intermediate neutrinos with path integrals [14]. Moreover, many treatments within the QFT framework were also proposed [15-21], aiming to solve the various unclear aspects of the quantum mechanics of neutrino oscillations $[10,13]$.

It has been known for a long time that the coherence necessary for neutrino oscillations depends crucially on localization aspects of the particles involved in the production of neutrinos [7]. This point of view can be

*Electronic address: ccnishi@ift.unesp.br supported by QFT arguments [19] as well. It raises then the question of how the coherent superposition of mass eigenstate neutrinos, which is called a "flavor" eigenstate, is created [20]. One way that became customary to avoid the ambiguities involving the question on how neutrinos are created and detected is to use an external (E) wave packet (WP) approach [17], in contrast to an intermediate (I) WP approach. According to Ref. [17], the IWP treatments are the simpler first quantized ones treating the propagation of neutrinos as free localized wave packets. In contrast, EWP approaches consider localized wave packets for the sources and detection particles while the neutrinos were considered intermediate virtual particles. The central issue distinguishing the general IWP and EWP approaches is: Despite its direct unobservability, is the intermediate neutrino a real (on-shell) particle propagating freely? If the answer is affirmative, the IWP approaches would be a good approximation of the oscillation phenomena.

On the other hand, another classification scheme can be used to classify the various existing treatments considering a more physical criterion irrespective of the use of WPs. Consider the descriptions of neutrino oscillations that (A) include explicitly the interactions responsible for the mixing and (B) those that only treat the propagation of neutrinos, i.e., . the mixing is an ad hoc ingredient. A more subtle aspect in between would be the (explicit or phenomenologically modeled) consideration of the production (and detection) process(es). In general, the IWP approaches are of type (B). The EWP approaches are of type (A). The BV approach, although in the QFT formalism, is of type (B) since mixing is introduced without 
explicitly including the interaction responsible for it. The type (B) approaches have the virtue that they can be formulated in a way in which total oscillation probability in time is always conserved and normalized to one [3,9]. This feature will be present in all first quantized approaches treated here (Secs. II and III and in a second quantized version (Sec. IVA). If different observables are considered, or a modeling of the details of the production and detection processes is attempted, further normalization is necessary $[4,8,12]$. In such cases, the oscillating observable might differ from the oscillation probability. On the other hand, type (A) approaches tend to be more realistic and can account for the production and detection processes giving experimentally observable oscillation probabilities [21]. Of course, they are essential to the investigation of how neutrinos are produced and detected $[11,20]$. We are not directly interested in these matters here.

Considering first quantized type (B) approaches, some recent works treating the flavor oscillation for spin one-half particles [22,23] have already found additional oscillatorial effects compared to usual oscillation formulas with WPs [8,9]. These effects are investigated and it is shown in Sec. II how these additional oscillatorial behaviors, which have characteristic frequencies much greater than usual oscillation frequencies, come from the interference between positive and negative frequency components of the initial WP. It can be understood as a consequence of the impossibility to simultaneously exclude all negative energy contributions of the initial spinorial wave function with respect to bases characterized by different masses. Moreover, these rapid oscillations are always present, independently of the initial WP, if a well-defined flavor is attributed to the initial WP.

To make clear the origin of the additional oscillatory contributions, we calculate, in Sec. III, the oscillation formula for a charged spin 0 particle in the Sakata-Taketani Hamiltonian formalism [24], which is equivalent to the Klein-Gordon scalar wave equation. (The explicit analysis with the mixed Klein-Gordon equation is made in Ref. [25], paying special attention to the relativistic initial value problem.) The oscillation formula in this case also possesses the additional interference terms between positive and negative frequency parts, very similar to the one obtained in the spin 1/2 case. From this example we will see that these interference terms are inevitable from a relativistic classical field theory perspective where covariance and causality is required. It is not specially associated to the spin degree of freedom.

Another particular ingredient of neutrino oscillations can be included naturally within Dirac theory: the lefthandedness of neutrinos created and detected through weak interactions. This fact, for a Dirac neutrino [26], implies an additional probability loss due to conversion of left-handed neutrinos into right-handed neutrinos, which is possible because chirality is no longer a constant of motion for massive Dirac particles [27]. Although previous calculations [23] have shown an approximate contribution to this effect, we calculate in Sec. II A the complete effect.

Concerning type (A) approaches, specifically the EWP description, we are interested to analyze further the propagation of intermediate virtual neutrinos. The framework where the investigations on first quantized approaches are made here is based on the calculation of the evolution kernels for free theories in presence of mixing. This enables us to deduce general oscillation probabilities in which there is explicit decoupling from the oscillating part (where all the oscillation information rests) and the initial wave packet. Another advantage of doing the calculations this way is that it resembles the propagator methods in covariant perturbation theory, which EWP approaches are based on. The free evolution kernel for fermions have a close relationship with the Feynman propagator used in QFT. What is common to both is that both particle and antiparticle parts contribute to the evolution or propagation. The necessity of the negative frequency part in the free evolution kernel is required from completeness and causality arguments but it also leads to the interference of positive and negative frequencies in flavor oscillation, treated in Secs. II and III. Then the question also arises in EWP approaches: Are there contributions from both particles and antiparticles in the propagation of virtual neutrinos? In a simple microscopic scattering process, this question is meaningless since virtual particles are usually off-shell particles and must naturally have both contributions. However, in EWP approaches the neutrinos propagate through macroscopic distances and, indeed, it can be shown $[19,20]$ that the virtual neutrinos are on-shell particles. There is no discussion, though, about the possibility of neutrino and antineutrino contributions to the process; both can be on-shell. This investigation is carried on in Sec. IV calculating explicitly the amplitude of production/ propagation/detection process in an EWP approach.

As a last task, we develop a simple, type (B), second quantized description of flavor oscillation in Sec. IVA using the free second quantized spin $1 / 2$ fermionic theory in the presence of mixing. This treatment has some similarities with the BV formalism but it does not require the introduction of flavor Fock spaces and Bogoliubov transformations. It means that the Fock space considered will be the one spanned by the mass eigenstates. Within this formalism it will be shown that the additional rapid oscillation contributions calculated through first quantized approaches do not survive the second quantization since only superpositions of particles (antiparticles) are used as initial neutrino (antineutrino) flavor states. Moreover, this property is not satisfied in the BV approach because the BV flavor states are mixtures of particle and antiparticle components; this is the ingredient responsible for a different oscillation probability [3]. 


\section{FLAVOR OSCILLATION FOR DIRAC FERMIONS}

It is well known that the Dirac equation can give a significantly good description of a Dirac fermion if its inherent localization is much bigger than its Compton wavelength; usually this is associated with weak external fields. For example, the spectrum for the hydrogen atom can be obtained with the relativistic corrections included (fine structure) [28], page 72. One of the terms responsible for fine structure, the Darwin term, can be interpreted as coming from the interference between positive and negative frequency parts (zitterbewegung) of the hydrogen eigenfunction in Dirac theory compared to the nonrelativistic theory [24]. On the other hand, a situation where the theory fails to give a satisfactory physical description is exemplified by the Klein paradox [28], page 62: The transmission coefficient for an electron moving towards a step barrier becomes negative for certain barrier heights, exactly when the localization of the electron wave function inside the barrier is comparable with its Compton wavelength.

Bearing in mind that first quantized approaches may fail under certain conditions, we will treat in this section the flavor oscillation problem using the free Dirac theory in the presence of two families mixing. The extension to treat three families of neutrinos is straightforward. A matricial notation will be used throughout the article for the first quantized approaches to express the mixing.

In matricial notation the mixing relation between flavor wave functions $\Psi_{f}^{\top}(\mathbf{x}) \equiv\left(\psi_{\nu_{e}}^{\top}(\mathbf{x}), \psi_{\nu_{\mu}}^{\top}(\mathbf{x})\right)$ and mass wave functions $\Psi_{m}^{\top}(\mathbf{x}) \equiv\left(\psi_{1}^{\top}(\mathbf{x}), \psi_{2}^{\top}(\mathbf{x})\right)$ is

$$
\Psi_{f}(\mathbf{x}) \equiv U \Psi_{m}(\mathbf{x})=\left(\begin{array}{cc}
\cos \theta & \sin \theta \\
-\sin \theta & \cos \theta
\end{array}\right) \Psi_{m}(\mathbf{x}) .
$$

Each mass wave function is defined as a four-component spinorial function $\psi_{n}(\mathbf{x}, t), n=1,2$ that satisfies the free Dirac equation

$$
i \frac{\partial}{\partial t} \psi_{n}(\mathbf{x}, t)=H_{n}^{D} \psi_{n}(\mathbf{x}, t), \quad n=1,2,
$$

where the free Hamiltonian is the usual

$$
H_{n}^{D} \equiv-i \boldsymbol{\alpha} \cdot \nabla+\beta m_{n}, \quad n=1,2 .
$$

We will work in the flavor diagonal basis. This choice defines the flavor basis vectors simply as

$$
\hat{\nu}_{e}^{\top}=(1,0), \quad \hat{\nu}_{\mu}^{\top}=(0,1),
$$

while the flavor projectors are obviously

$$
\mathrm{P}_{\nu_{\alpha}} \equiv \hat{\nu}_{\alpha} \hat{\nu}_{\alpha}^{\top}
$$

Actually, as an abuse of notation, the equivalence $U \sim U \otimes$ $\mathbb{1}_{D}$ is implicit, as well as $\mathrm{P}_{\nu_{\alpha}} \sim \mathrm{P}_{\nu_{\alpha}} \otimes \mathbb{1}_{D}$; the symbol $\mathbb{1}_{D}$ refers to the identity matrix in spinorial space.

The total Hamiltonian governing the dynamics of $\Psi_{m}$ is $H^{D}=\operatorname{diag}\left(H_{1}^{D}, H_{2}^{D}\right)$. From the considerations above, $\Psi_{f}(\mathbf{x}, t)$ satisfy the equation

$$
i \frac{\partial}{\partial t} \Psi_{f}(\mathbf{x}, t) \equiv U H^{D} U^{-1} \Psi_{f}(\mathbf{x}, t) .
$$

The solution to the equation above can be written in terms of a flavor evolution operator $K^{D}$ as

$$
\begin{aligned}
\Psi_{f}(\mathbf{x}, t) & =K^{D}(t) \Psi_{f}(\mathbf{x}, 0) \\
& =\int d^{3} \mathbf{x}^{\prime} K^{D}\left(\mathbf{x}-\mathbf{x}^{\prime} ; t\right) \Psi_{f}\left(\mathbf{x}^{\prime}, 0\right),
\end{aligned}
$$

where

$$
K^{D}\left(\mathbf{x}-\mathbf{x}^{\prime} ; t\right)=\int \frac{d^{3} \mathbf{p}}{(2 \pi)^{3}} K^{D}(\mathbf{p} ; t) e^{i \mathbf{p} \cdot\left(\mathbf{x}-\mathbf{x}^{\prime}\right)} .
$$

We can calculate $K^{D}(t)$ in any representation (momentum or position) as

$$
K^{D}(t)=U e^{-i H^{D} t} U^{-1}=\left(\begin{array}{cc}
\cos ^{2} \theta e^{-i H_{1}^{D} t}+\sin ^{2} \theta e^{-i H_{2}^{D} t} & -\cos \theta \sin \theta\left(e^{-i H_{1}^{D} t}-e^{-i H_{2}^{D} t}\right) \\
-\cos \theta \sin \theta\left(e^{-i H_{1}^{D} t}-e^{-i H_{2}^{D} t}\right) & \sin ^{2} \theta e^{-i H_{1}^{D} t}+\cos ^{2} \theta e^{-i H_{2}^{D} t}
\end{array}\right) .
$$

The conversion probability is then

$$
\begin{aligned}
\mathcal{P}\left(\nu_{\mathrm{e}} \rightarrow \nu_{\mu} ; t\right) & =\int d \mathbf{x} \Psi_{f}^{\dagger}(\mathbf{x}, 0) K^{D \dagger}(t) \mathrm{P}_{\nu_{\mu}} K^{D}(t) \Psi_{f}(\mathbf{x}, 0) \\
& =\int d \mathbf{p} \tilde{\psi}_{\nu_{e}}^{\dagger}(\mathbf{p})\left(K_{\mu e}^{D}\right)^{\dagger} K_{\mu e}^{D}(\mathbf{p}, t) \tilde{\psi}_{\nu_{e}}(\mathbf{p}),(10)
\end{aligned}
$$

satisfying the initial condition $\Psi_{f}^{\top}(\mathbf{x}, 0)=\left(\psi_{\nu_{e}}^{\top}(\mathbf{x}, 0), 0\right)$. Such an initial condition implies, in terms of mass eigenfunctions, $\psi_{1}(\mathbf{x}, 0)=\psi_{2}(\mathbf{x}, 0)=\psi_{\nu_{e}}(\mathbf{x})$, as a requirement to obtain an initial wave function with definite flavor [9]. The function $\tilde{\psi}_{\nu_{e}}(\mathbf{p})$ denotes the inverse Fourier transform of $\psi_{\nu_{e}}(\mathbf{x})$ [see Eqs. (A1) and (A2)].

Before obtaining the conversion probability for Dirac fermions, let us replace the spinorial functions $\psi_{n}(\mathbf{x})$ by spinless one-component wave functions $\varphi_{n}(\mathbf{x})$ in the flavor wave function $\Psi_{f}^{\top}(\mathbf{x}) \rightarrow\left(\varphi_{\nu_{e}}(\mathbf{x}), \varphi_{\nu_{\mu}}(\mathbf{x})\right)$ and mass wave function $\Psi_{m}^{\top}(\mathbf{x}) \rightarrow\left(\varphi_{1}(\mathbf{x}), \varphi_{2}(\mathbf{x})\right)$. We also replace the Dirac Hamiltonian in momentum space $H_{n}^{D}(\mathbf{p})$ (3) by the relativistic energy $E_{n}(\mathbf{p})=\sqrt{\mathbf{p}^{2}+m_{n}^{2}}$. Inserting these replacements into Eq. (10) we can recover the usual oscillation probability $[9,23]$

$$
\begin{aligned}
\mathcal{P}\left(\nu_{e} \rightarrow \nu_{\mu} ; t\right) & =\int d \mathbf{x}\left|\hat{\nu}_{\mu}^{\top} \Psi_{f}(\mathbf{x}, t)\right|^{2} \\
& =\int d \mathbf{p}\left|K_{\mu e}^{S}(\mathbf{p}, t) \tilde{\varphi}_{\nu_{e}}(\mathbf{p})\right|^{2} \\
& =\int d \mathbf{p} \mathcal{P}(\mathbf{p}, t)\left|\tilde{\varphi}_{\nu_{e}}(\mathbf{p})\right|^{2},
\end{aligned}
$$


where $\quad \Psi_{f}(\mathbf{x}, 0)^{\top}=\left(\varphi_{\nu_{e}}(\mathbf{x})^{\top}, 0\right), \quad K_{\mu e}^{S}(\mathbf{p}, t) \equiv\left(K^{S}\right)_{21}=$ $-\sin \theta \cos \theta\left(e^{-i E_{1}(\mathbf{p}) t}-e^{-i E_{2}(\mathbf{p}) t}\right)$ and

$$
\mathcal{P}(\mathbf{p}, t)=\sin ^{2} 2 \theta \sin ^{2}(\Delta E(\mathbf{p}) t / 2)
$$

is just the standard oscillation formula. The conversion probability (11) in this case is then the standard oscillation probability smeared out by the initial momentum distribution. If the substitution $\left|\tilde{\varphi}_{\nu_{e}}(\mathbf{p})\right|^{2} \rightarrow \delta^{3}\left(\mathbf{p}-\mathbf{p}_{0}\right)$ is made, the standard oscillation formula is recovered: It corresponds to the plane-wave limit.

After we have checked the standard oscillation formula can be recovered for spinless particles restricted to positive energies in the plane-wave limit, we can return to the case of Dirac fermions. We can obtain explicitly the terms of the mixed evolution kernel (9) by using the property of the Dirac Hamiltonian in momentum space $H_{n}^{D^{2}}=E_{n}^{2}(\mathbf{p}) \mathbb{1}_{D}$, which leads

$$
\begin{aligned}
\left(K_{\mu e}^{D}\right)^{\dagger} K_{\mu e}^{D}(\mathbf{p}, t)= & \mathcal{P}(\mathbf{p}, t)[1-f(\mathbf{p})] \mathbb{1}_{D} \\
& +\sin ^{2} 2 \theta f(\mathbf{p}) \sin ^{2}(\bar{E} t) \mathbb{1}_{D},
\end{aligned}
$$

where

$$
f(\mathbf{p})=\frac{1}{2}\left[1-\frac{\mathbf{p}^{2}+m_{1} m_{2}}{E_{1} E_{2}}\right],
$$

and $\mathcal{P}(\mathbf{p}, t)$ is the standard conversion probability function (12). A unique implication of Eq. (13), which is proportional to the identity matrix in spinorial space, is that the conversion probability (10) does not depend on the spinorial structure of the initial flavor wave function but only on its momentum density as

$$
\begin{aligned}
\mathcal{P}\left(\nu_{\mathrm{e}} \rightarrow \nu_{\mu} ; t\right)= & \int d \mathbf{p}\{\mathcal{P}(\mathbf{p}, t)[1-f(\mathbf{p})] \\
& \left.+\sin ^{2} 2 \theta f(\mathbf{p}) \sin ^{2}(\bar{E} t)\right\} \tilde{\psi}_{\nu_{e}}^{\dagger}(\mathbf{p}) \tilde{\psi}_{\nu_{e}}(\mathbf{p}) .
\end{aligned}
$$

(The tilde will denote the inverse Fourier transformed function throughout this paper.) Furthermore, the modifications in Eq. (15) compared to the scalar conversion probability (11) are exactly the same modifications found in Refs. [22,23] after smearing out through a specific Gaussian wave packet.

The conservation of total probability

$$
\mathcal{P}\left(\nu_{e} \rightarrow \nu_{\mu} ; t\right)+\mathcal{P}\left(\nu_{e} \rightarrow \nu_{e} ; t\right)=1,
$$

is automatic in virtue of

$$
K_{e e}^{D \dagger}(\mathbf{p}, t) K_{e e}^{D}(\mathbf{p}, t)+K_{\mu e}^{D \dagger}(\mathbf{p}, t) K_{\mu e}^{D}(\mathbf{p}, t)=\mathbb{1}_{D},
$$

and the survival and conversion probability for an initial muon neutrino are identical to the probabilities for an initial electron neutrino because of the relations

$$
\begin{aligned}
& K_{\mu \mu}^{D \dagger}(\mathbf{p}, t) K_{\mu \mu}^{D}(\mathbf{p}, t)=K_{e e}^{D \dagger}(\mathbf{p}, t) K_{e e}^{D}(\mathbf{p}, t), \\
& K_{\mu e}^{D \dagger}(\mathbf{p}, t) K_{\mu e}^{D}(\mathbf{p}, t)=K_{e \mu}^{D \dagger}(\mathbf{p}, t) K_{e \mu}^{D}(\mathbf{p}, t) .
\end{aligned}
$$

To explain the origin of the additional oscillatory terms in Eq. (15) it is instructive to rewrite the free Dirac time evolution operator, in momentum space, in the form

$$
e^{-i H_{n}^{D} t}=e^{-i E_{n} t} \Lambda_{n+}^{D}+e^{i E_{n} t} \Lambda_{n-}^{D},
$$

where

$$
\Lambda_{n \pm}^{D}=\frac{1}{2}\left(\mathbb{1}_{D} \pm \frac{H_{n}^{D}}{E_{n}}\right)
$$

are the projector operators to positive $(+)$ or negative ( ) energy eigenstates of $H_{n}^{D}$. By using the decomposition above (20), we can analyze $K_{\mu e}^{D}$ in Eq. (9), which contains the terms

$$
\begin{aligned}
e^{i H_{1}^{D} t} e^{-i H_{2}^{D} t}= & e^{i \Delta E t} \Lambda_{1+}^{D} \Lambda_{2+}^{D}+e^{-i \Delta E t} \Lambda_{1-}^{D} \Lambda_{2-}^{D} \\
& +e^{i 2 \bar{E} t} \Lambda_{1+}^{D} \Lambda_{2-}^{D}+e^{-i 2 \bar{E} t} \Lambda_{1-}^{D} \Lambda_{2+}^{D},
\end{aligned}
$$

plus its Hermitian conjugate. Since $\Lambda_{1 \pm}^{D} \Lambda_{2 \mp}^{D} \neq 0$, it can be seen that the rapid oscillating terms come from the interference between, e.g., the positive frequencies of the Hamiltonian $H_{1}^{D}$ and negative energies of the Hamiltonian $H_{2}^{D}$. One may think that by restricting the initial wave function to contain only positive energy contributions would eliminate the rapid oscillatory terms, as zitterbewegung disappears for superpositions of solely positive energy states in Dirac theory [28], but it does not happen. The positive energy eigenfunctions with respect to a basis characterized by a mass $m_{1}$ necessarily have nonnull components of negative energy with respect to another basis characterized by $m_{2}$ (this point is illustrated in Appendix B). Thus the rapid oscillatory contributions are an inevitable consequence of this framework and it is always present independently of the initial WP, if initially a definite flavor is chosen. However, its influence, quantified by the function $f(\mathbf{p})$ in Eq. (14), is negligible for momentum distributions around ultrarelativistic values [22]. These rapid oscillatory terms will also be found for charged spin 0 particle oscillations in the next section, with contributions slightly different from the ones obtained for spin $1 / 2$ particles.

\section{A. Inclusion of left handedness}

Until this point, we have been considering the oscillation of general flavor "particle number" for general Dirac neutrinos. However, due to the left-handed nature of weak interactions only left-handed components are produced and detected. To incorporate this fact into, for example, the conversion probability in Eq. (10), it is sufficient to use initial left-handed WPs and replace the kernel of Eq. (13) by the projected counterpart 


$$
\begin{aligned}
L K_{\mu e}^{D \dagger}(\mathbf{p}, t) L K_{\mu e}^{D}(\mathbf{p}, t) L= & \mathcal{P}^{D}(\mathbf{p}, t) L-\frac{1}{4} \sin ^{2} 2 \theta \\
& \times\left(\frac{m_{1}}{E_{1}} \sin E_{1} t-\frac{m_{2}}{E_{2}} \sin E_{2} t\right)^{2} L,
\end{aligned}
$$

where $\mathcal{P}^{D}(\mathbf{p}, t)=K_{\mu e}^{D \dagger}(\mathbf{p}, t) K_{\mu e}^{D}(\mathbf{p}, t)$ is the conversion kernel of Eq. (13) and $L=\left(1-\gamma_{5}\right) / 2$ is the projector to left chirality. The conservation of total probability (16) no longer holds because there is a probability loss due to the undetected right-handed component,

$$
L K_{\mu e}^{D \dagger} R K_{\mu e}^{D}(\mathbf{p}, t) L=\frac{1}{4} \sin ^{2} 2 \theta\left(\frac{m_{1}}{E_{1}} \sin E_{1} t-\frac{m_{2}}{E_{2}} \sin E_{2} t\right)^{2} L,
$$

where $R=\left(1+\gamma_{5}\right) / 2$ is the projector to right chirality. We can see that the probability loss $(24)$ is proportional to the ratio $m_{n}^{2} / E_{n}^{2}$ which is negligible for ultrarelativistic neutrinos. The total probability loss for an initial lefthanded electron neutrino turning into right-handed neutrinos, irrespective of the final flavor, is given by the kernel

$$
\begin{aligned}
& L K_{\mu e}^{D \dagger} R K_{\mu e}^{D}(\mathbf{p}, t) L+L K_{e e}^{D \dagger} R K_{e e}^{D}(\mathbf{p}, t) L \\
& \quad=\left[\cos ^{2} \theta\left(\frac{m_{1}}{E_{1}}\right)^{2} \sin ^{2} E_{1} t+\sin ^{2} \theta\left(\frac{m_{2}}{E_{2}}\right)^{2} \sin ^{2} E_{2} t\right] L .
\end{aligned}
$$

To obtain the unphysical complementary kernels responsible for the conversion of right-handed component to right-handed and left-handed components, it is enough to make the substitution $L \leftrightarrow R$ in all formulas.

\section{FLAVOR OSCILLATION FOR SPIN 0}

The derivation of the usual conversion probability (11) takes into account only the positive frequency contributions. The mass wave function used to obtain Eq. (11) corresponds to the solutions of the wave equation

$$
i \frac{\partial}{\partial t} \varphi(\mathbf{x}, t)=\sqrt{-\nabla^{2}+m^{2}} \varphi(\mathbf{x}, t),
$$

which is equivalent to the Dirac equation in the FoldyWouthuysen representation [29], restricted to positive energies. The evolution kernel for this equation is not satisfactory from the point of view of causality [30], page 18, i.e., the kernel is not null for spacelike intervals. Moreover, the eigenfunctions restricted to one sign of energy do not form a complete set [24].

To recover a causal propagation in the spin 0 case, the Klein-Gordon wave equation must be considered. In the first quantized version, the spectrum of the solutions have positive and negative energy as in the Dirac case. However, to take advantage of the Hamiltonian formalism used so far, it is more convenient to work in the Sakata-Taketani (ST) Hamiltonian formalism [24] where each mass wave function is formed by two components,

$$
\Phi_{n}(\mathbf{x}, t)=\left(\begin{array}{c}
\varphi_{n}(\mathbf{x}, t) \\
\chi_{n}(\mathbf{x}, t)
\end{array}\right), \quad n=1,2
$$

The components $\varphi$ and $\chi$ are combinations of the usual scalar Klein-Gordon wave function $\phi(x)$ and its time derivative $\partial_{0} \phi(x)$. This is necessary since the Klein-Gordon equation is a second order differential equation in time and the knowledge of the function and its time derivative is necessary to completely define the time evolution.

The time evolution in this formalism is governed by the Hamiltonian [24]

$$
H_{n}^{\mathrm{ST}}=-\left(\tau_{3}+i \tau_{2}\right) \frac{\nabla^{2}}{2 m_{n}}+m_{n}^{2}
$$

which satisfies the condition $\left(H_{n}^{\mathrm{ST}}\right)^{2}=\left(-\nabla^{2}+m_{n}^{2}\right) \mathbb{1}_{\mathrm{ST}}$, like the Dirac Hamiltonian (3). The $\tau_{k}$ represents the usual Pauli matrices and $\mathbb{1}_{\mathrm{ST}}$ is the identity matrix.

A charge density [31] can be defined as

$$
\bar{\Phi}_{n} \Phi_{n} \equiv \Phi_{n}^{\dagger} \tau_{3} \Phi_{n}=\left|\varphi_{n}\right|^{2}-\left|\chi_{n}\right|^{2},
$$

which is equivalent to the one found in Klein-Gordon notation $i \phi^{*} \overleftrightarrow{\partial}_{0} \phi$. Needless to say, this density (29) is only non-null for complex (charged) wave functions. The charge density $\bar{\Phi} \Phi$ is the equivalent of fermion probability density $\psi^{\dagger} \psi$ in the Dirac case, although the former is not positive definite as the latter. The adjoint $\bar{\Phi}=\Phi^{\dagger} \tau_{3}$ were defined to make explicit the (nonpositive definite) norm structure of the conserved charge,

$$
\int d \mathbf{x} \bar{\Phi}_{n}(\mathbf{x}, t) \Phi_{n}(\mathbf{x}, t) \equiv\left(\Phi_{n}, \Phi_{n}\right)=\text { time independent. }
$$

Consequently, the adjoint of any operator $\Omega$ can be defined as $\bar{\Omega}=\tau_{3} \Omega^{\dagger} \tau_{3}$, satisfying $(\bar{\Omega} \Phi, \Phi)=(\Phi, \Omega \Phi)$. Within this notation, the Hamiltonians of Eq. (28) are self-adjoint, $\bar{H}_{n}^{\mathrm{ST}}=H_{n}^{\mathrm{ST}}$, and the time invariance of Eq. (30) is assured.

We can assemble, as in the previous section, the mass wave functions into $\Psi_{m}^{\top} \equiv\left(\Phi_{1}^{\top}, \Phi_{2}^{\top}\right)$ and the flavor wave functions into $\Psi_{f}^{\top} \equiv\left(\Phi_{\nu_{e}}^{\top}, \Phi_{\nu_{\mu}}^{\top}\right)$, satisfying the mixing relation $\Psi_{f} \equiv U \Psi_{m}$. The equivalence of $U \sim U \otimes \mathbb{1}_{\mathrm{ST}}$ and of $\mathrm{P}_{\nu_{\alpha}} \sim \mathrm{P}_{\nu_{\alpha}} \otimes \mathbb{1}_{\mathrm{ST}}$ is implicit without modification in the notations. Then, the time evolution of $\Psi_{f}$ can be given through a time evolution operator $K^{\mathrm{ST}}$ acting in the same form as in Eq. (7). In complete analogy to the calculations from Eq. (8)-(11), we can define the conversion probability as

$$
\begin{aligned}
\mathcal{P}\left(\nu_{e} \rightarrow \nu_{\mu} ; t\right) & =\int d \mathbf{x} \bar{\Psi}_{f}(\mathbf{x}, 0) \overline{K^{\mathrm{ST}}(t)} \mathrm{P}_{\nu_{\mu}} K^{\mathrm{ST}}(t) \Psi_{f}(\mathbf{x}, 0) \\
& =\int d \mathbf{p} \overline{\tilde{\Phi}_{e}(\mathbf{p})} \overline{K_{\mu e}^{\mathrm{ST}}} K_{\mu e}^{\mathrm{ST}}(\mathbf{p}, t) \tilde{\Phi}_{e}(\mathbf{p})
\end{aligned}
$$

where $\Psi_{f}(\mathbf{x}, 0)^{\top}=\left(\Phi_{e}(\mathbf{x})^{\top}, 0\right)$. The adjoint operation was 
also extended to $\bar{\Psi}_{f}=\Psi_{f}^{\dagger}\left(\mathbb{1}_{\theta} \otimes \tau_{3}\right)$, where $\mathbb{1}_{\theta}$ is the identity in mixing space.

The information of time evolution, hence oscillation, is all encoded in

$$
\begin{aligned}
\overline{K_{\mu e}^{\mathrm{ST}}} K_{\mu e}^{\mathrm{ST}}(\mathbf{p}, t)= & \mathcal{P}(\mathbf{p}, t)[1-f(\mu \mathbf{p})] \mathbb{1}_{\mathrm{ST}} \\
& +\sin ^{2} 2 \theta f(\mu \mathbf{p}) \sin ^{2}(\bar{E} t) \mathbb{1}_{\mathrm{ST}},
\end{aligned}
$$

where the function $f(\mathbf{p})$ was already defined in Eq. (14) and

$$
\mu=\sqrt{\frac{1}{2}\left(\frac{m_{1}}{m_{2}}+\frac{m_{2}}{m_{1}}\right)} .
$$

The factor $\mu \geq 1$ determines the difference with the Dirac case in Eq. (13). The equality $\mu=1$ holds when $m_{1}=m_{2}$, i.e., when there is no oscillation.

\section{CONNECTION WITH QUANTUM FIELD THEORY}

The main improvement of the covariant approaches developed in Secs. II and III is that the propagation kernels governed by Dirac and Sakata-Taketani Hamiltonians are causal, i.e., are null for spacelike separations [see Eqs. (A18) and (A19) and Refs. [28,30,32] ]. On the contrary, the kernel of spinless particles restricted only to positive energies is not null for spacelike intervals [30]. From the point of view of relativistic classical field theories, a causal kernel guarantees, by the Cauchy theorem, the causal connection between the wave function in two spacelike surfaces at different times [32].

To compare the IWP and EWP approaches, it is useful to rewrite the Dirac evolution kernel for a fermion of mass $m_{n}$, present in Eq. (7), in the form [28], page 89,

$$
\begin{aligned}
K_{n}^{D}(x-y)= & \sum_{s} \int \frac{d^{3} p}{2 E_{n}}\left[u_{n}^{s}(x ; \mathbf{p}) \bar{u}_{n}^{s}(y ; \mathbf{p})\right. \\
& \left.+v_{n}^{s}(x ; \mathbf{p}) \bar{v}_{n}^{s}(y ; \mathbf{p})\right] \gamma_{0} \\
\equiv & i S\left(x-y ; m_{n}\right) \gamma_{0}, \\
n= & 1,2,
\end{aligned}
$$

where $(x-y)^{0}=t,(x-y)^{i}=\left(\mathbf{x}-\mathbf{x}^{\prime}\right)^{i}$ when compared to the notation of Eq. (7). The spinorial functions $u, v$, are the free solutions of the Dirac equation and they are explicitly defined in Appendix A. (More familiar forms for the function $S$ are also shown in Appendix A.) Clearly the function $i S\left(x-y ; m_{n}\right)=\left\langle 0\left|\left\{\psi_{n}(x), \bar{\psi}_{n}(y)\right\}\right| 0\right\rangle$ satisfies the homogeneous Dirac equation with mass $m_{n}(2)$ and it is known to be null for spacelike intervals $(x-y)^{2}<0$ $[30,32]$.

In contrast, the Feynman propagator $i S_{F}(x-y)$ appears in QFT. It is a Green function for the inhomogeneous Dirac equation obeying particular boundary conditions. The EWP approaches use this Green function for the propaga- tion of virtual neutrinos. To directly compare the Feynman propagator to the kernel in Eq. (34), we can write $i S_{F}$ in the form

$$
\begin{aligned}
i S_{F}\left(x-y ; m_{n}\right) \equiv & \left\langle 0\left|T\left(\psi_{n}(x), \bar{\psi}_{n}(y)\right)\right| 0\right\rangle \\
= & \sum_{s} \int \frac{d^{3} p}{2 E_{n}}\left[u_{n}^{s}(x ; \mathbf{p}) \bar{u}_{n}^{s}(y ; \mathbf{p}) \theta\left(x_{0}-y_{0}\right)\right. \\
& \left.-v_{n}^{s}(x ; \mathbf{p}) \bar{v}_{n}^{s}(y ; \mathbf{p}) \theta\left(y_{0}-x_{0}\right)\right] .
\end{aligned}
$$

Although the function $S_{F}$ is called causal propagator, it is not null for spacelike intervals, and it naturally arises in QFT when interactions are present and treated in a covariant fashion. Equation (35) shows that the propagator $S_{F}$ describes positive energy states propagating forward in time and negative energy states propagating backward in time [28], page 91. At a first glance, both neutrino and antineutrino parts of Eq. (35) seem to contribute to the space-time integrations present in covariant perturbation theory, as neutrino-antineutrino contributions in Eq. (34) have led to Eq. (15).

In the following we will show in an EWP approach that for large separations between production and detection both neutrino and antineutrino parts may contribute as intermediate neutrinos for certain situations.

We will follow the calculations made in Ref. [20], using, instead of the scalar interaction, the effective chargedcurrent weak Lagrangian

$$
\begin{gathered}
\mathcal{L}_{W}=G \sum_{i, \alpha=1}^{N=3}\left[\bar{l}_{\alpha}(x) \gamma^{\mu} L U_{\alpha i} \nu_{i}(x) J_{\mu}(x)\right. \\
\left.+\bar{\nu}_{i}(x) U_{\alpha i}^{*} \gamma^{\mu} L l_{\alpha}(x) J_{\mu}^{\dagger}(x)\right] \\
=\mathcal{L}_{1}+\mathcal{L}_{1}^{\dagger},
\end{gathered}
$$

where $G=\sqrt{2} G_{F}$ and $J_{\mu}$ is the sum of any effective leptonic or hadronic current. The Lagrangian (36) is written only in terms of physical mass eigenstate fields, which coincides with flavor eigenstate fields only for the charged leptons: $l_{1}(x) \equiv e(x), l_{2}(x) \equiv \mu(x), \ldots$

Suppose the process $[20,33]$ where a charged lepton $l_{\alpha}$ hit a nucleus $A$ turning it into another nucleus $A^{\prime}$ with emission of a neutrino (this process happens around $x_{A}$ ). Subsequently the neutrino travels a long distance and hit a nucleus $B$ which transforms into $B^{\prime}$ emitting a lepton $l_{\beta}$ (this process happens around $x_{B}$ ). The whole process looks like $l_{\alpha}+A+B \rightarrow l_{\beta}+A^{\prime}+B^{\prime}$ with transition amplitude given by

$$
\left\langle A^{\prime}\left(\mathbf{p}_{A}^{\prime}\right), B^{\prime}\left(\mathbf{p}_{B}^{\prime}\right), l_{\beta}\left(\mathbf{p}_{\beta}\right)|S| A, B, l_{\alpha}\right\rangle .
$$

The final states are momentum eigenstates while the initial states are localized [20]. The lowest order nonzero contribution of the scattering matrix $S$ to Eq. (38) is second order in the Lagrangian (36). More explicitly, the term that contributes to the amplitude (38) comes from 


$$
\begin{gathered}
S^{(2)}=\frac{i^{2}}{2} T\left\langle\mathcal{L}_{W}\right\rangle^{2}=-\frac{1}{2} T\left\langle\mathcal{L}_{1}+\mathcal{L}_{1}^{\dagger}\right\rangle^{2} \\
\sim-T\left\langle\mathcal{L}_{1}\right\rangle\left\langle\mathcal{L}_{1}^{\dagger}\right\rangle \\
\sim-G^{2} \int d^{4} x d^{4} y \sum_{\beta \alpha} \mathcal{L}_{\beta \alpha}(x, y),
\end{gathered}
$$

where \langle\rangle stands for space-time integration and

$$
\begin{array}{r}
\mathcal{L}_{\beta \alpha}(x, y) \equiv \sum_{i}: J_{\mu}(x) \bar{l}_{\beta}(x) \gamma^{\mu} L U_{\beta i} i S_{F}(x \\
\left.-y ; m_{i}\right) U_{\alpha i}^{*} \gamma^{\nu} L l_{\alpha}(y) J_{\nu}^{\dagger}(y):
\end{array}
$$

In Eq. (40) we kept only the mixed product and in Eq. (41) we kept from all possible terms in Wick expansion [28], page 180 , only the term responsible for the transition of interest.

Then the transition amplitude (38) can be calculated as

$$
\begin{aligned}
-G^{-2}\left\langle A^{\prime}\left(\mathbf{p}_{A}^{\prime}\right), B^{\prime}\left(\mathbf{p}_{B}^{\prime}\right), l_{\beta}\left(\mathbf{p}_{\beta}\right)\left|S^{(2)}\right| A, B, l_{\alpha}\right\rangle \\
=\int d^{4} y d^{4} x\left\langle B^{\prime}\left(\mathbf{p}_{B}^{\prime}\right)\left|J_{\mu}(y)\right| B\right\rangle \\
\quad \times\left\langle A^{\prime}\left(\mathbf{p}_{A}^{\prime}\right)\left|J_{\nu}^{\dagger}(x)\right| A\right\rangle \bar{u}_{\beta}\left(y, \mathbf{p}_{\beta}\right) \gamma^{\mu} L \\
\quad \times \sum_{i} U_{\beta i} U_{\alpha i}^{*} i S_{F}\left(y-x ; m_{i}\right) \gamma^{\nu} L\left\langle 0\left|l_{\alpha}(x)\right| l_{\alpha}\right\rangle \\
\equiv \sum_{i} U_{\beta i} U_{\alpha i}^{*} \mathcal{A}_{i} .
\end{aligned}
$$

The initial states must be chosen in such a way that $A, l_{\alpha}$ are localized around $x_{A}=\left(t_{A}, \mathbf{x}_{A}\right)$ and $B$ is localized around $x_{B}=\left(t_{B}, \mathbf{x}_{B}\right)$, since we are ultimately interested in large separations $\left|\mathbf{x}_{B}-\mathbf{x}_{A}\right|$. We can implement explicitly those localization conditions into the wave packets

$$
\begin{aligned}
\left\langle B^{\prime}\left(\mathbf{p}_{B}^{\prime}\right)\left|J_{\mu}(y)\right| B\right\rangle= & \frac{1}{(2 \pi)^{3 / 2}} \int \widehat{d \mathbf{q}_{B}} e^{i p_{B}^{\prime} \cdot y} J_{\mu}^{B B^{\prime}}\left(\mathbf{q}_{B}, \mathbf{p}_{B}^{\prime}\right) \\
& \times \psi_{B}\left(\mathbf{q}_{B}\right) e^{-i q_{B} \cdot\left(y-x_{B}\right)} \\
\left\langle A^{\prime}\left(\mathbf{p}_{A}^{\prime}\right)\left|J_{\nu}^{\dagger}(x)\right| A\right\rangle= & \frac{1}{(2 \pi)^{3 / 2}} \int \widehat{d \mathbf{q}_{A}} e^{i p_{A}^{\prime} \cdot y} J_{\mu}^{A A^{\prime}}\left(\mathbf{q}_{A}, \mathbf{p}_{A}^{\prime}\right) \\
& \times \psi_{A}\left(\mathbf{q}_{A}\right) e^{-i q_{A} \cdot\left(x-x_{A}\right)} \\
\left\langle 0\left|l_{\alpha}(x)\right| l_{\alpha}\right\rangle= & \frac{1}{(2 \pi)^{3 / 2}} \int \widehat{d \mathbf{q}_{\alpha}} \psi_{\alpha}\left(\mathbf{q}_{\alpha}\right) e^{-i q_{\alpha} \cdot\left(x-x_{A}\right)},
\end{aligned}
$$

where $\quad \widehat{d \mathbf{q}}=d \mathbf{q}(2 E(\mathbf{q}))^{-1 / 2}, \quad J_{\mu}^{B B^{\prime}}\left(\mathbf{q}_{B}, \mathbf{p}_{B}^{\prime}\right)=$ $\left\langle B^{\prime}\left(\mathbf{p}_{B}^{\prime}\right)\left|J_{\mu}(0)\right| B\left(\mathbf{q}_{B}\right)\right\rangle \quad$ and $\quad J_{\nu}^{A A^{\prime}}\left(\mathbf{q}_{A}, \mathbf{p}_{A}^{\prime}\right)=$ $\left\langle A^{\prime}\left(\mathbf{p}_{A}^{\prime}\right)\left|J_{\nu}^{\dagger}(0)\right| A\left(\mathbf{q}_{A}\right)\right\rangle$.

Following the calculations from Eq. (43) with the localization aspects of Eqs. (45)-(47) included, we arrive at

$$
\begin{aligned}
\mathcal{A}_{i}= & \frac{1}{(2 \pi)^{6}} \int \widehat{d \mathbf{q}_{B}} \int \widehat{d \mathbf{q}_{A}} \int \widehat{d \mathbf{q}_{\alpha}} J_{\mu}^{B B^{\prime}}\left(\mathbf{q}_{B}, \mathbf{p}_{B}^{\prime}\right) \psi_{B}\left(\mathbf{q}_{B}\right) \\
& \times J_{\mu}^{A A^{\prime}}\left(\mathbf{q}_{A}, \mathbf{p}_{A}^{\prime}\right) \psi_{A}\left(\mathbf{q}_{A}\right) e^{i q_{B} \cdot x_{B}} e^{i\left(q_{A}+q_{\alpha}\right) \cdot x_{A}} \bar{u}_{\beta}\left(\mathbf{p}_{\beta}\right) \\
& \times \gamma^{\mu} L\left[\int d^{4} x d^{4} y e^{i \kappa_{\beta} \cdot y} e^{-i \kappa_{\alpha} \cdot x} i S_{i}(y-x)\right] \gamma^{\nu} L \psi_{\alpha}\left(\mathbf{q}_{\alpha}\right),
\end{aligned}
$$

where $\kappa_{\beta}=\left(\kappa_{\beta}^{0}, \boldsymbol{\kappa}_{\beta}\right), \kappa_{\alpha}=\left(\kappa_{\alpha}^{0}, \boldsymbol{\kappa}_{\alpha}\right)$, and

$$
\begin{aligned}
\boldsymbol{\kappa}_{\beta} & \equiv \mathbf{p}_{\beta}+\mathbf{p}_{B}^{\prime}-\mathbf{q}_{B}, \\
\boldsymbol{\kappa}_{\beta}^{0} & \equiv E_{\beta}\left(\mathbf{p}_{\beta}\right)+E_{B^{\prime}}\left(\mathbf{p}_{B}^{\prime}\right)-E_{B}\left(\mathbf{q}_{B}\right), \\
\boldsymbol{\kappa}_{\alpha} & \equiv \mathbf{p}_{\alpha}-\mathbf{p}_{A}^{\prime}+\mathbf{q}_{A}, \\
\boldsymbol{\kappa}_{\alpha}^{0} & \equiv E_{\alpha}\left(\mathbf{q}_{\alpha}\right)-E_{A^{\prime}}\left(\mathbf{p}_{A}^{\prime}\right)+E_{A}\left(\mathbf{q}_{A}\right) .
\end{aligned}
$$

By using the results of Eqs. (C1) and (C2), the expression between square brackets in Eq. (48) gives

$$
\begin{aligned}
2 \pi \delta\left(\kappa_{\beta}^{0}\right. & \left.-\kappa_{\alpha}^{0}\right) \int d \mathbf{x} d \mathbf{y} \frac{-i}{4 \pi r} e^{i k_{\omega} r} e^{-i \boldsymbol{\kappa}_{\beta} \cdot \mathbf{y}} \\
& \times e^{i \boldsymbol{\kappa}_{\alpha} \cdot \mathbf{x}}\left[u_{i}\left(k_{\omega} \hat{\mathbf{r}}\right) \bar{u}_{i}\left(k_{\omega} \hat{\mathbf{r}}\right) \theta\left(\omega_{i}-m_{i}\right)\right. \\
& \left.-v_{i}\left(-k_{\omega} \hat{\mathbf{r}}\right) \bar{v}_{i}\left(-k_{\omega} \hat{\mathbf{r}}\right) \theta\left(-\omega_{i}-m_{i}\right)\right],
\end{aligned}
$$

where $r \equiv|\mathbf{y}-\mathbf{x}|, \hat{\mathbf{r}} \equiv(\mathbf{y}-\mathbf{x}) / r, \omega_{i} \equiv \kappa_{\beta}^{0}=\kappa_{\alpha}^{0}$, and $k_{\omega} \equiv \sqrt{\omega_{i}^{2}-m_{i}^{2}}$. The crucial point here is that, depending on the masses and momenta of the incoming particles, both neutrinos $(u \bar{u})$ and antineutrinos $(v \bar{v})$ can contribute to the amplitude (48) depending on the sign of its energy $\omega_{i}$, restricted to $\left|\omega_{i}\right|>m_{i}$; the off-shell contributions for $\omega_{i} \in$ $\left[-m_{i}, m_{i}\right]$ are exponentially decreasing and then negligible for large distances (see Appendix C). We will see in the following that antineutrino contributions in this case are possible and it corresponds to unphysical contributions.

We are interested in large production-detection separations. It permits us to approximate, as in Ref. [20], $r \approx$ $R+\hat{\mathbf{R}} \cdot\left(\mathbf{y}-\mathbf{x}_{B}\right)-\hat{\mathbf{R}} \cdot\left(\mathbf{x}-\mathbf{x}_{A}\right)$ and $\hat{\mathbf{r}} \approx \hat{\mathbf{R}}$, where $R \equiv$ $\left|\mathbf{x}_{B}-\mathbf{x}_{A}\right|$ and $\hat{\mathbf{R}} \equiv\left(\mathbf{x}_{B}-\mathbf{x}_{A}\right) / R$. Such approximations inserted in Eq. (51) lead to momentum conservation on $x_{A}$ and $x_{B}$ vertices:

$$
\begin{aligned}
2 \pi \delta\left(\kappa_{\beta}^{0}-\kappa_{\alpha}^{0}\right) \frac{-i}{4 \pi R} e^{i k_{\omega} R} e^{-i k_{\omega} \hat{\mathbf{R}} \cdot\left(\mathbf{x}_{B}-\mathbf{x}_{A}\right)}(2 \pi)^{3} \delta^{3}\left(\kappa_{\beta}-k_{\omega} \hat{\mathbf{R}}\right) \\
\quad \times(2 \pi)^{3} \delta^{3}\left(\kappa_{\alpha}-k_{\omega} \hat{\mathbf{R}}\right)\left[u_{i}\left(k_{\omega} \hat{\mathbf{R}}\right) \bar{u}_{i}\left(k_{\omega} \hat{\mathbf{R}}\right) \theta\left(\omega_{i}-m_{i}\right)\right. \\
\left.-v_{i}\left(-k_{\omega} \hat{\mathbf{R}}\right) \bar{v}_{i}\left(-k_{\omega} \hat{\mathbf{R}}\right) \theta\left(-\omega_{i}-m_{i}\right)\right] .
\end{aligned}
$$

At this point we have all the information to analyze whether the antineutrino part of the propagator contributes to the overall process. Neither of the isolated processes $A+l_{\alpha} \rightarrow A^{\prime}+\bar{\nu}_{i}$ and $B+\bar{\nu}_{i} \rightarrow B^{\prime}+l_{\beta}$ are allowed if we calculate the transition amplitude for them separately using the weak Lagrangian (36). (For Majorana neutrinos they are strongly suppressed by helicity mismatch.) So far fourmomentum conservation in both $x_{A}$ and $x_{B}$ vertices were 
automatically required from the calculations; among them the requirement of energy conservation for intermediate neutrinos with respect to the accompanying particles in vertex $x_{A}\left(\omega_{i}=\kappa_{\alpha}^{0}\right)$ and in vertex $x_{B}\left(\omega_{i}=\kappa_{\beta}^{0}\right)$, is already implicit. The remaining are explicit in the delta functions of Eq. (52). The on-shell condition for neutrinos $\left(\left|\omega_{i}\right|^{2}-\right.$ $k_{\omega}^{2}=m_{i}^{2}$ ) for long distance propagation was also automatic. What the calculations did not required is a definite sign for $\omega_{i}$, for all possible momenta constrained by the mentioned energy-momentum conservations. To analyze if and under what conditions both signs are possible is equivalent to study the kinematics of two-body to twobody scattering allowing the sign of one particle energy to be free. Putting in equations, for vertex $x_{A}$, assuming the particle $A$ at rest, we obtain from $\left(p_{A}-p_{i}\right)^{2}=$ $\left(p_{A^{\prime}}-p_{\alpha}\right)^{2}$ the neutrino energy

$$
\begin{aligned}
E_{i}= & \frac{1}{2 M_{A}}\left[M_{A}^{2}-M_{A^{\prime}}^{2}+m_{i}^{2}-m_{\alpha}^{2}\right. \\
& \left.+2 E_{\alpha} E_{A^{\prime}}-2 \mathbf{p}_{\alpha} \cdot \mathbf{p}_{A}^{\prime}\right] .
\end{aligned}
$$

The minimum value of the right-hand side of Eq. (53) corresponds to the last two terms equal to $2 m_{\alpha} M_{A^{\prime}}$, which gives for the minimum

$$
\min \left(E_{i}\right)=\frac{1}{2 M_{A}}\left[M_{A}^{2}-\left(M_{A^{\prime}}-m_{\alpha}\right)^{2}+m_{i}^{2}\right] .
$$

The values $\omega_{i}=\kappa_{\alpha}^{0}$ are bounded from below by the value in Eq. (54). Imposing $\min \left(E_{i}\right)>m_{i}$ and $\min \left(E_{i}\right)<-m_{i}$ is, respectively, equivalent to

$$
\begin{aligned}
& M_{A}-M_{A^{\prime}}>-\left(m_{\alpha}-m_{i}\right) \\
& M_{A}-M_{A^{\prime}}<-\left(m_{\alpha}+m_{i}\right),
\end{aligned}
$$

for $M_{A}>m_{i}$ and $M_{A^{\prime}}>m_{\alpha}$. It is clear that, depending on the value of the masses, condition (56) may be satisfied leading to antineutrino contributions to Eq. (48) for a range of possible incoming momenta. Of course the condition (55) is sufficient to exclude antineutrino contributions but it also excludes the cases where a threshold energy is required for the lepton $l_{\alpha}$ to initiate the production reaction. Thus to prevent antineutrino contributions, it is better to adopt the weaker condition of restricting the sign of the energy of intermediate neutrinos $\omega_{i}$ to be positive, keeping only the first term in Eq. (51). Analogous analysis leads to possible momenta and mass values that allow $\kappa_{\beta}^{0}<-m_{i}$ for vertex $x_{B}$, still compatible with $\kappa_{\alpha}^{0}=\kappa_{\beta}^{0}$. Notice that condition $\kappa_{\alpha}^{0}>m_{i}$ is exactly the kinematical condition to allow the production of physical neutrinos in $x_{A}$ and $\kappa_{\beta}^{0}>$ $m_{i}$ allow only the contribution of neutrinos with energy above threshold to trigger the detection reaction. The violation of these conditions implies in kinematically impossible contributions in production or detection.

Restricted to condition $\omega_{i}>0$ we can insert the expression above into Eq. (48) which yields

$$
\begin{aligned}
\mathcal{A}_{i}= & \int \widehat{d \mathbf{q}_{\alpha}} 2 \pi \delta\left(\kappa_{\beta}^{0}-\kappa_{\alpha}^{0}\right) \theta\left(\omega_{i}-m_{i}\right) \\
& \times \frac{-i}{4 \pi R} e^{i k_{\omega} R-i \omega_{i}\left(t_{B}-t_{A}\right)} e^{i\left(p_{k}+p_{B}^{\prime}\right) \cdot x_{B}} e^{i p_{A}^{\prime} \cdot x_{A}} u_{k}\left(\mathbf{p}_{\beta}\right) \\
& \times \gamma^{\mu} L u_{i}\left(k_{\omega} \hat{\mathbf{R}}\right) \bar{u}_{i}\left(k_{\omega} \hat{\mathbf{R}}\right) \gamma^{\nu} L \psi_{\alpha}\left(\mathbf{q}_{\alpha}\right) J_{\mu}^{B B^{\prime}}\left(\mathbf{q}_{B}, \mathbf{p}_{B}^{\prime}\right) \\
& \times\left.\frac{\psi_{B}\left(\mathbf{q}_{B}\right)}{\sqrt{E_{B}\left(\mathbf{q}_{B}\right)}} J_{\nu}^{A A^{\prime}}\left(\mathbf{q}_{A}, \mathbf{p}_{A}^{\prime}\right) \frac{\psi_{A}\left(\mathbf{q}_{A}\right)}{\sqrt{E_{A}\left(\mathbf{q}_{A}\right)}}\right|_{\substack{\mathbf{q}_{B}=\mathbf{p}_{\beta}+\mathbf{p}_{B}^{\prime}-k_{\omega} \hat{\mathbf{R}} \\
\mathbf{q}_{A}=\mathbf{p}_{A}^{\prime}-\mathbf{q}_{\alpha}+k_{\omega} \mathbf{R}}}
\end{aligned}
$$

Notice that the step function $\theta\left(\omega_{i}-m_{i}\right)$ prevents nonphysical neutrinos to contribute to the process.

Particularly, if we use a unidimensional wave packet for the incoming lepton $l_{\alpha}$

$$
\psi_{\alpha}(\mathbf{q})=\psi_{\alpha}\left(q_{x}, q_{y}, q_{z}\right)=\delta\left(q_{x}\right) \delta\left(q_{y}\right) \psi_{\alpha z}\left(q_{z}\right),
$$

we obtain an amplitude analogous to Ref. [20]:

$$
\begin{aligned}
& \sum_{i} U_{\beta i} U_{\alpha i}^{*} \mathcal{A}_{i}=\sum_{i} \frac{-i}{4 \pi R} e^{i k_{\omega} R-i \omega_{i}\left(t_{B}-t_{A}\right)} 2 \pi\left|2 \mathrm{p}_{\alpha} \frac{\partial}{\partial \mathbf{q}_{\alpha}^{2}}\left(\kappa_{\beta}^{0}-\kappa_{\alpha}^{0}\right)\right|_{\mathbf{q}_{\alpha}=\mathrm{p}_{\alpha} \hat{z}}^{-1} e^{i\left(p_{k}+p_{B}^{\prime}\right) \cdot x_{B}} e^{i p_{A}^{\prime} \cdot x_{A}} U_{\beta i} u_{\beta}\left(\mathbf{p}_{\beta}\right)
\end{aligned}
$$

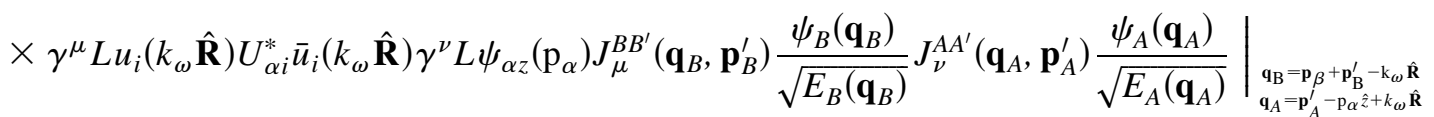

where $\mathrm{p}_{\alpha}$ is the root of $f\left(\left|\mathbf{q}_{\alpha}\right|=\mathrm{p}_{\alpha}\right)=\kappa_{\beta}^{0}-\kappa_{\alpha}^{0}=0$, which comes from energy conservation from the whole process; if there is no root the process is kinematically forbidden. The detection probability is proportional to the square of the amplitude (59) integrated over the final phase space $d \mathbf{p}_{A}^{\prime} d \mathbf{p}_{B}^{\prime} d \mathbf{p}_{\beta}\left[2 E_{A^{\prime}}\left(\mathbf{p}_{A}^{\prime}\right) 2 E_{B^{\prime}}\left(\mathbf{p}_{B}^{\prime}\right) 2 E_{\beta}\left(\mathbf{p}_{\beta}\right)\right]^{-1}$. In particular, since $\mathbf{p}_{\beta}, \mathbf{p}_{A}^{\prime}, \mathbf{p}_{B}^{\prime}$ are fixed, the phases that differ for different intermediate neutrinos $\nu_{i}$ are only $k_{\omega} R-$ $\omega_{i}\left(t_{A}-t_{B}\right)$ which is the same result obtained in
Ref. [20] (except for terms which depend on the mean velocity of particles $A$ and $B$ ).

So far we have shown in an EWP approach both processes in $x_{A}$ and $x_{B}$ should be considered real scattering processes with real neutrinos involved. The off-shell contributions are negligible to large distances and antineutrino contributions were explicitly excluded by eliminating the second term of Eq. (51). This information permits us to rewrite Eq. (57) in a slightly different form: 


$$
\begin{aligned}
-G^{2} \sum_{i} U_{\beta i} U_{\alpha i}^{*} \mathcal{A}_{i}= & \sum_{i} \int \frac{d \mathbf{p}}{2 E_{i}(\mathbf{p})} \int d^{4} y\left\langle B^{\prime}\left(\mathbf{p}_{B}^{\prime}\right), l_{\beta}\left(\mathbf{p}_{\beta}\right)\right| \\
& \times \mathcal{L}_{1}(y) e^{i\left(P-p_{i}\right) \cdot x_{B}}\left|B, \nu_{i}(\mathbf{p})\right\rangle \\
& \times \int d^{4} x \theta(y-x)\left\langle A^{\prime}\left(\mathbf{p}_{A}^{\prime}\right), \nu_{i}(\mathbf{p})\right| \\
& \times \mathcal{L}_{1}^{\dagger}(x) e^{i P \cdot x_{A}}\left|A, l_{\alpha}\right\rangle,
\end{aligned}
$$

where $P=(H, \mathbf{P})$ is the energy-momentum operator. A change of notation were made here: in Eq. (59) the states $|B\rangle$ and $\left|A, l_{\alpha}\right\rangle$ are centered around the origin while in Eqs. (38)-(47) they are, respectively, centered around $x_{B}$ and $x_{A}$; the translation is explicitly performed by the translation operator $e^{i P \cdot x}$. Additionally, the step function $\theta(y-$ $x$ ) is necessary to ensure that the contributions of points $y$ around $x_{B}$ should always be after the contributions of points $x$ around $x_{A}$. By following the same steps from Eq. (43) to Eq. (58) we can arrive from Eq. (60) to (59).

Equation (60) shows us the amplitude of the entire process from production to detection in "decomposed" form (apart from the step function in time): the amplitude of production process multiplied by the amplitude of detection process summed over all possible intermediate real neutrinos of different masses $m_{i}$ and momentum p. (The sum over spins are implicit.)

\section{A. A simple second quantized formulation}

Considering that only real neutrinos or antineutrinos (one of them exclusively) should travel from production to detection, the possibility to use the free second quantized theory for spin $1 / 2$ fermions to describe flavor oscillations is investigated in this section. This simple, type $B$ and QFT description of flavor oscillation phenomena guarantees only particle or antiparticle propagation, keeping the nice property of giving normalized oscillation probabilities, like the first quantized examples treated in Secs. II and III.

To accomplish the task of calculating oscillation probabilities in QFT, we have to define the neutrino states that are produced and detected through weak interactions. First, we define the shorthand for the combination of fields appearing in the weak effective charged-current Lagrangian (36)

$$
\nu_{\alpha}(x) \equiv U_{\alpha i} \nu_{i}(x), \quad \alpha=e, \mu .
$$

We will restrict the problem to two flavor families and use the matrix $U$ as the same in Eq. (1). The mass eigenfields $\nu_{i}(x), i=1,2$, are the physical fields for which the mass eigenstates $\left|\nu_{i}(\mathbf{p})\right\rangle$ are well-defined asymptotic states. The free fields $\nu_{i}(x)$ can be expanded in terms of creation and annihilation operators (see Appendix A) and the projection to the one-particle space defines the mass wave function,

$$
\begin{aligned}
\psi_{\nu_{i}}\left(\mathbf{x} ; g_{i}\right) & =\left\langle 0\left|\nu_{i}(\mathbf{x})\right| \nu_{i}: g_{i}\right\rangle \equiv \sum_{s} \int d \mathbf{p} \frac{g_{i}^{S}(\mathbf{p})}{\sqrt{2 E_{i}}} u_{i}^{s}(\mathbf{x} ; \mathbf{p}), \\
i & =1,2,
\end{aligned}
$$

where

$$
\left|\nu_{i}: g_{i}\right\rangle \equiv \sum_{s} \int d \mathbf{p} g_{i}^{s}(\mathbf{p})\left|\nu_{i}(\mathbf{p}, s)\right\rangle
$$

Since the creation operators for neutrinos (antineutrinos) can be written in terms of the free fields $\bar{\nu}_{i}(x)\left[\nu_{i}(x)\right]$, we can define the flavor states as the superpositions of mass eigenstates

$$
\left|\nu_{\alpha}:\{g\}\right\rangle \equiv U_{\alpha i}^{*}\left|\nu_{i}: g_{i}\right\rangle \quad\left|\bar{\nu}_{\alpha}:\{g\}\right\rangle \equiv U_{\alpha i}\left|\bar{\nu}_{i}: g_{i}\right\rangle .
$$

The details of creation are encoded in the functions $g_{i}$.

We can also define

$$
\psi_{\nu_{\alpha} \nu_{e}}(x ;\{g\}) \equiv\left\langle 0\left|\nu_{e}(x)\right| \nu_{\alpha}:\{g\}\right\rangle=U_{e i} U_{\alpha i}^{*} \psi_{\nu_{i}}\left(x ; g_{i}\right),
$$

where $\psi_{\nu_{i}}(x)$ are then mass wave functions defined in Eq. (62). We can see from Eq. (65) that if $\psi_{\nu_{1}}(\mathbf{x}, t)=$ $\psi_{\nu_{2}}(\mathbf{x}, t)=\psi(\mathbf{x})$, for a given time $t, \psi_{\nu_{e} \nu_{e}}(\mathbf{x}, t)=\psi(\mathbf{x})$ and $\psi_{\nu_{\mu} \nu_{e}}(\mathbf{x}, t)=0$ due to the unitarity of the mixing matrix.

Although this approach does not rely on flavor Fock spaces and Bogoliubov transformations, we can use the same observables used by Blasone and Vitiello to quantify flavor oscillation [34]: the flavor charges, which are defined as

$$
Q_{\alpha}(t)=\int d \mathbf{x}: \nu_{\alpha}^{\dagger}(\mathbf{x}, t) \nu_{\alpha}(\mathbf{x}, t):, \quad \alpha=e, \mu,
$$

where : : denotes normal ordering. Note that the $Q_{e}(t)+$ $Q_{\mu}(t)=Q$ is conserved [3], the two flavor charges are compatible for equal times, i.e., $\left[Q_{e}(t), Q_{\mu}(t)\right]=0$, and $\langle\nu:\{g\}|Q| \nu:\{g\}\rangle= \pm\langle\nu:\{g\} \mid \nu:\{g\}\rangle$ for any particle state $(+$ ) or antiparticle state $(-)$. Notice that in the second quantized version the charges can acquire negative values, despite the fermion probability density in first quantization is a positive definite quantity. The conservation of total charge guarantees the conservation of total probability (16).

We can further split the flavor charges into left-handed $(-)$ and right-handed $(+)$ parts,

$$
\begin{aligned}
Q_{\alpha}^{( \pm)}(t) & =\int d \mathbf{x}: \nu_{\alpha}^{\dagger}(\mathbf{x}, t) \frac{1}{2}\left(\mathbb{1} \pm \gamma_{5}\right) \nu_{\alpha}(\mathbf{x}, t):, \\
\alpha & =e, \mu,
\end{aligned}
$$

where $Q_{\alpha}^{(+)}+Q_{\alpha}^{(-)}=Q_{\alpha}$. These components will be used to calculate the left-handed to right-handed transition.

With the flavor charges defined, we can calculate, for example, the conversion probability 


$$
\begin{gathered}
\mathcal{P}\left(\nu_{e} \rightarrow \nu_{\mu} ; t\right) \equiv\left\langle\nu_{e}:\{g\}\left|Q_{\mu}(t)\right| \nu_{e}:\{g\}\right\rangle \\
=U_{\mu i} U_{\mu j}^{*} U_{e j} U_{e i}^{*} \int d \mathbf{p} e^{-i\left(E_{i}-E_{j}\right) t} \tilde{\psi}_{\nu_{j}}^{\dagger}\left(\mathbf{p} ; g_{j}\right) \tilde{\psi}_{\nu_{i}}\left(\mathbf{p} ; g_{i}\right),
\end{gathered}
$$

where the neutrino wave functions $\psi_{\nu_{i}}$ are defined in terms of the function $g_{i}(\mathbf{p})$ in Eq. (62). If we could equate the two mass wave functions in momentum space $\tilde{\psi}_{\nu_{1}}\left(\mathbf{p} ; g_{1}\right)=$ $\tilde{\psi}_{\nu_{2}}\left(\mathbf{p} ; g_{2}\right)=\tilde{\psi}_{\nu_{e}}(\mathbf{p})$ we would obtain, from Eq. (69), the standard two family conversion probability (11)

$$
\mathcal{P}\left(\nu_{e} \rightarrow \nu_{\mu} ; t\right)=\int d \mathbf{p} \mathcal{P}(\mathbf{p}, t) \tilde{\psi}_{\nu_{e}}^{\dagger}(\mathbf{p}) \tilde{\psi}_{\nu_{e}}(\mathbf{p}),
$$

where $\mathcal{P}$ was defined in Eq. (12). However, the equality cannot hold as proved in Appendix B: Two wave functions with only positive energy components with respect to two bases characterized by different masses cannot be equal. Then, it is not possible to impose a flavor definite condition. Instead, we can write

$$
g_{i}(\mathbf{p}, s)=\frac{u_{i}^{s \dagger}(\mathbf{p})}{\sqrt{2 E_{i}(\mathbf{p})}} \tilde{\psi}_{i}(\mathbf{p}),
$$

where $\tilde{\psi}_{i}(\mathbf{p})$ is the initial wave function associated to the neutrino of mass $m_{i}$ at creation, taking care to maintain the normalization $\int d \mathbf{p}\left|g_{i}(\mathbf{p})\right|^{2}=1$; any transition amplitude can be written in the form Eq. (71). In general $\tilde{\psi}_{i}(\mathbf{p})=$ $\tilde{\psi}\left(\mathbf{p}, m_{i}\right)$, and then, for small mass differences,

$$
\tilde{\psi}_{i}(\mathbf{p}) \approx \tilde{\psi}(\mathbf{p}, \bar{m}) \pm \frac{\Delta m}{2} \frac{\partial}{\partial \bar{m}} \tilde{\psi}(\mathbf{p}, \bar{m})
$$

where $\bar{m}=\left(m_{1}+m_{2}\right) / 2$ and $\Delta m=m_{2}-m_{1}$. Keeping only the first term, $\tilde{\psi}(\mathbf{p}, \bar{m}) \equiv \tilde{\psi}(\mathbf{p})$, we obtain from Eq. (69),

$$
\begin{aligned}
\mathcal{P}\left(\nu_{e} \rightarrow \nu_{\mu} ; t\right)= & \int d \mathbf{p} \mathcal{P}(\mathbf{p}, t) \tilde{\psi}^{\dagger}(\mathbf{p})\left[\mathbb{1}-\frac{1}{2} \Lambda_{1-}(\mathbf{p})\right. \\
& \left.-\frac{1}{2} \Lambda_{2-}(\mathbf{p})\right] \tilde{\psi}(\mathbf{p})+\frac{1}{4} \sin ^{2} 2 \theta \int d \mathbf{p} \tilde{\psi}^{\dagger}(\mathbf{p}) \\
& \times\left[f(\mathbf{p}) \cos (\Delta E t)-i \frac{\Delta m}{2 E_{1} E_{2}} \boldsymbol{\gamma}\right. \\
& \cdot \mathbf{p} \sin (\Delta E t)] \tilde{\psi}(\mathbf{p}) .
\end{aligned}
$$

Notice that, in this case, the conversion probability is nonnull for $t=0$,

$$
\mathcal{P}\left(\nu_{e} \rightarrow \nu_{\mu} ; 0\right)=\frac{1}{4} \sin ^{2} 2 \theta \int d \mathbf{p} f(\mathbf{p}) \tilde{\psi}^{\dagger}(\mathbf{p}) \tilde{\psi}(\mathbf{p}),
$$

which implies a direct lepton flavor violation in creation. However, since $f(\mathbf{p}) \approx(\Delta m)^{2} /\left(4 \mathbf{p}^{2}\right)$ for ultrarelativistic momenta, the violation is hopelessly feeble for direct measurement. Among the deviations of the conversion probability (73) compared to the standard one (70), only the last term is of order $\Delta m / \bar{E}$, the rest is of order $(\Delta m / \bar{E})^{2}$ [the contributions of $\Lambda_{i-}$ can be estimated by $\left[\boldsymbol{v}_{2}^{\dagger}(-\mathbf{p}, s) u_{1}\left(\mathbf{p}, s^{\prime}\right)\right]^{2} \sim \mathbf{p}^{2}[\Delta m+\Delta E]^{2} /\left[\left(m_{1}+E_{1}\right)\left(m_{1}+\right.\right.$ $\left.\left.E_{1}\right)\right]$ ]. Even so, $\Delta m / \bar{E} \sim 10^{-9}$ for $\Delta m^{2} \sim 10^{-3} \mathrm{eV}^{2}, \bar{m} \sim$ $1 / 2 \mathrm{eV}$, and $\bar{E} \sim 1 \mathrm{MeV}$, which cannot be seen in actual oscillation experiments. Nevertheless, it is important to note that the knowledge of $\Delta m$ in conjunction with $\Delta m^{2}$ gives information about the absolute mass scale because of $\Delta m^{2}=2 \bar{m} \Delta m$.

Using $Q_{\alpha}^{(-)}$of Eq. (67) instead of $Q_{\alpha}$ in Eq. (69) and $\tilde{\psi}(\mathbf{p})=L \tilde{\psi}(\mathbf{p})$ in Eq. (70) we obtain

$$
\begin{aligned}
\mathcal{P}\left(\nu_{e L} \rightarrow \nu_{\mu R} ; t\right)= & \int d \mathbf{p}\left[\frac{m_{1} m_{2}}{4 E_{1} E_{2}} \mathcal{P}(\mathbf{p}, t)\right. \\
& \left.+\frac{1}{4} \sin ^{2} 2 \theta\left(\frac{m_{1}}{2 E_{1}}-\frac{m_{2}}{2 E_{2}}\right)^{2}\right] \tilde{\psi}^{\dagger}(\mathbf{p}) \tilde{\psi}(\mathbf{p}) .
\end{aligned}
$$

The total probability loss from the conversion of initial left-handed electron neutrino to right-handed neutrinos yields

$$
\begin{aligned}
\mathcal{P}\left(\nu_{e L} \rightarrow\right. & \left.\nu_{e R} ; t\right)+\mathcal{P}\left(\nu_{e L} \rightarrow \nu_{\mu R} ; t\right) \\
= & \int d \mathbf{p}\left[\cos ^{2} \theta\left(\frac{m_{1}}{2 E_{1}}\right)^{2}\right. \\
& \left.+\sin ^{2} \theta\left(\frac{m_{2}}{2 E_{2}}\right)^{2}\right] \tilde{\psi}^{\dagger}(\mathbf{p}) \tilde{\psi}(\mathbf{p}) .
\end{aligned}
$$

Notice Eq. (76) does not depend on time in contrast to its first quantized analog in Eq. (25). Other conversion and survival probabilities can be obtained from Eq. (17) and

$$
\mathcal{P}\left(\nu_{e L} \rightarrow \nu_{\mu R} ; t\right)+\mathcal{P}\left(\nu_{e L} \rightarrow \nu_{\mu L} ; t\right)=\mathcal{P}\left(\nu_{e} \rightarrow \nu_{\mu} ; t\right)
$$

The exchange of $L \leftrightarrow R$ does not modify the formulas, provided we also change the chirality of the initial wave function.

For completeness we calculate the additional conversion probabilities including the second term of Eq. (72)

$$
\begin{aligned}
\delta \mathcal{P}\left(\nu_{e} \rightarrow \nu_{\mu} ; t\right)= & \frac{1}{4} \sin ^{2} 2 \theta \frac{\Delta m}{2} \int d \mathbf{p} \tilde{\psi}^{\dagger}(\mathbf{p})\left[\frac{H_{2}}{2 E_{2}}-\frac{H_{1}}{2 E_{1}}\right. \\
& +\frac{\Delta m}{2 E_{1} E_{2}} \boldsymbol{\gamma} \cdot \mathbf{p}+\left(\Lambda_{1+}+\Lambda_{2+}\right. \\
& -f(\mathbf{p})) i \sin \Delta E t] \frac{\partial}{\partial \bar{m}} \tilde{\psi}(\mathbf{p})+\text { H.c. }
\end{aligned}
$$




$$
\begin{aligned}
\delta \mathcal{P}\left(\nu_{e L} \rightarrow \nu_{\mu R} ; t\right)= & \frac{1}{4} \sin ^{2} 2 \theta \frac{\Delta m}{2} \int d \mathbf{p} \tilde{\psi}^{\dagger}(\mathbf{p})\left[\left(\frac{m_{2}}{2 E_{2}}\right)^{2}\right. \\
& \left.-\left(\frac{m_{1}}{2 E_{1}}\right)^{2}+\frac{m_{1} m_{2}}{2 E_{1} E_{2}} i \sin \Delta E t\right] \\
& \times \frac{\partial}{\partial \bar{m}} \tilde{\psi}(\mathbf{p})+\text { H.c. },
\end{aligned}
$$

which have terms of order $\Delta m$ and $(\Delta m)^{2}$.

To calculate the conversion probability for antineutrinos $\bar{\nu}_{e} \rightarrow \bar{\nu}_{\mu}$, it is enough to use

$$
g_{i}^{s *}(\mathbf{p}) \equiv \tilde{\psi}_{i}^{\dagger}(\mathbf{p}) \frac{v_{i}^{s}(\mathbf{p})}{\sqrt{2 E_{i}(\mathbf{p})}},
$$

instead of Eq. (71), replace $t \rightarrow-t$ and $\tilde{\psi}(\mathbf{p}) \rightarrow \frac{\alpha \cdot \mathbf{p}}{|\mathbf{p}|} \tilde{\psi}(\mathbf{p})$ in the expressions corresponding to neutrinos (69)-(79), or apply charge conjugation $\tilde{\psi}(\mathbf{p}) \rightarrow-\gamma_{0} C \tilde{\psi}^{*}(\mathbf{p})$. These prescriptions can be inferred from direct comparison to the calculations and beware that the definition of antineutrino states are defined with $g_{i}^{s *}(\mathbf{p})$ (A29).

The formulas obtained in this second quantized version do not have the interference terms between positive and negative energies like in Eq. (15). Such interference terms are absent because the possible mixed terms like $b_{2}(\mathbf{p}) a_{1}^{\dagger}(\mathbf{p})|0\rangle$ are null for an initial flavor state superposition that contains only particle states (or only antiparticles states). Notice that the irrelevance of the initial spinorial structure no longer holds in this second quantized version, which can be seen, for example, in Eq. (73).

\section{DISCUSSION AND CONCLUSIONS}

Using the Dirac equation which is a relativistic covariant equation we obtained oscillation probabilities respecting causal propagation. The oscillation formulas obtained had additional rapid oscillating terms depending on the frequency $E_{1}+E_{2}$, with respect to the usual oscillation formulas with wave packets. Such additional oscillatorial character seemed to have its origin in the intrinsic spinorial character used. However, we have seen that such terms also appear in the charged spin 0 particle oscillations. In fact, the rapid oscillatorial terms arise from the interference of positive and negative frequency parts of the initial WP and they are always present independently of the initial wave packet if initially a flavor definite condition were imposed. In addition, within Dirac theory, we have shown the detailed spinorial character of the initial wave function was irrelevant for flavor oscillation, independently of the momenta involved. The inclusion of the left-handed nature of the created and detected neutrinos could also be simply achieved. It is important to stress that the modifications found in this context would have tiny observable effects to the flavor oscillation of ultrarelativistic neutrinos.

Regarding second quantized approaches (Sec. IV), in particular, EWP approaches, we can compare the propagators used in the latter to the evolution kernels in IWP approaches. Both the free evolution kernel and the Feynman propagator for fermions contain the contribution from particles and antiparticles. From this perspective, EWP approaches could also contain both contributions from neutrinos and antineutrinos, as in the first quantized approaches presented in Secs. II and III. To analyze this point, an EWP calculation was carried out explicitly in Sec. IV following Ref. [20]. Restricted to a case where only neutrinos would be present, the calculation showed that the antineutrino contribution was not excluded automatically in the formalism but a subsidiary condition could be necessary: The sign of the frequency of the intermediate neutrinos should be restricted to be positive. In such a case, there can be interference terms between positive and negative frequencies, possibly yielding rapid oscillation terms similar to the ones obtained in Dirac theory of Sec. II. However, it should be stressed that the origin of the interference between positive and negative terms is different in the first quantized Dirac theory treated in Sec. II and in the EWP (second quantized) treated in this Sec. IV. The former comes from causality and completeness arguments in a classical field theory perspective, while the latter has its origin in the consideration of nonphysical contributions. The restriction implied by the subsidiary condition automatically guarantees that: (i) only real neutrinos that are kinematically allowed in production contributes and (ii) in detection, only neutrinos with energies above threshold to trigger the detection reaction contribute. Otherwise, kinematically forbidden reactions in production or detection could be possible through exchange of virtual antineutrinos carrying negative energy. The overall energy-momentum conservation, though, is always respected (smeared out through finite momentum distributions) through production/propagation/detection processes. Since the presence of both neutrino-antineutrino contributions is common to all EWP approaches, the subsidiary condition necessary in the EWP approach analyzed is possibly necessary in any approach with virtual neutrino propagation. [Unless a stronger condition like Eq. (55) is already implicit.] For example, in Eq. (14) of Ref. [19], the subsidiary condition (for antineutrinos) is satisfied because the detection reaction is an elastic scattering. [Although the detection electrons are off-shell (bound state), the subsidiary condition is valid.] An important remark in this respect is that the calculations of the production and detection amplitudes as separate processes take automatically into account the physical kinematical conditions (i) and (ii) through the energy-momentum delta functions. It is also important to stress that the results obtained here depended neither on particular WPs nor on the particular interaction used. The interesting point is that by imposing such subsidiary conditions beforehand enables us to write the transition amplitude for the entire production/propagation/detection process in a decomposed form, with simple physical interpretation. A more detailed discussion about the decompo- 
sition of the process in separate production, propagation, and detection processes can be found in Ref. [21]. Similar conclusions can be drawn for the case where only antineutrinos should propagate: The sign to be chosen should be negative. A realistic EWP approach for antineutrino propagation is given in Ref. [19]. To conclude this part, EWP approaches for neutrino oscillations require for consistency, but do not automatically imply, real intermediate neutrinos or antineutrinos exclusively.

All the properties discussed above about EWP approaches suggest that the description of two macroscopically distant scattering processes (production and detection) as a single scattering process described by a single scattering matrix has to be treated with care. We can be confident about the use of the $S$ matrix to describe any microscopic event through perturbation theory to any order of expansion (if the theory is renormalizable), but the extension to macroscopically distant reactions is not automatic. Actually, if the two processes are indeed not causally connected it can be proved that the $S$ matrix decomposes as the product of the two $S$ matrices for the two distant and independent processes [35].

From the considerations above, the positive and negative interference terms in the first quantized approaches considered seem unphysical. To support this idea, it was shown in Sec. IVA that a simple second quantized, type B, and IWP-like, approach could be devised using the second quantized free theory maintaining the simple properties of IWP approaches but eliminating the undesirable interference of positive and negative frequencies that was inevitable in the relativistic quantum mechanical context. On the other hand, new ingredients such as the direct flavor violation in creation and deviations from the standard oscillation formula were found. The deviations include the probability loss due to the conversion of left-handed neutrinos to right-handed neutrinos. Unfortunately, those new effects are tiny either because of the small mass difference or the ultrarelativistic nature of neutrinos and they are not feasible for direct observation in actual oscillation experiments.

\section{ACKNOWLEDGMENTS}

This work was supported by Conselho Nacional de Desenvolvimento Científico e Tecnológico (CNPq). The author would like to thank Professor J. C. Montero for a critical reading of the manuscript.

\section{APPENDIX A: NOTATION AND DEFINITIONS}

The (scalar, spinorial, or ST) wave functions related by Fourier transforms are denoted as

$$
\varphi(\mathbf{x})=\frac{1}{(2 \pi)^{3 / 2}} \int d \mathbf{p} \tilde{\varphi}(\mathbf{p}) e^{i \mathbf{p} \cdot \mathbf{x}}
$$

$$
\tilde{\varphi}(\mathbf{p})=\frac{1}{(2 \pi)^{3 / 2}} \int d \mathbf{x} \varphi(\mathbf{x}) e^{-i \mathbf{p} \cdot \mathbf{x}} .
$$

The tilde denotes the inverse Fourier transformed function.

Using the property of the Dirac or ST Hamiltonian, $H_{n}^{2}=\left(\mathbf{p}^{2}+m_{n}\right)^{2} \mathbb{1}$, we can write the evolution operator in the form

$$
e^{-i H_{n} t}=\cos \left(E_{n} t\right)-i \frac{H_{n}}{E_{n}} \sin \left(E_{n} t\right),
$$

where the momentum dependence have to be replaced by $-i \nabla$ in coordinate space.

The free neutrino field expansion used is $(i=1,2)$

$$
\nu_{i}(x)=\sum_{s} \int \frac{d \mathbf{p}}{2 E_{\mathbf{p}}}\left[u_{i}^{s}(x ; \mathbf{p}) a_{i}(\mathbf{p}, s)+v_{i}^{s}(x ; \mathbf{p}) b_{i}^{\dagger}(\mathbf{p}, s)\right],
$$

where the creation and annihilation operators satisfy the canonical anticommutation relations

$$
\begin{aligned}
& \left\{a_{i}(\mathbf{p}, r), a_{j}^{\dagger}\left(\mathbf{p}^{\prime}, s\right)\right\}=\delta_{i j} \delta_{r s} 2 E_{i}(\mathbf{p}) \delta^{3}\left(\mathbf{p}-\mathbf{p}^{\prime}\right), \\
& \left\{b_{i}(\mathbf{p}, r), b_{j}^{\dagger}\left(\mathbf{p}^{\prime}, s\right)\right\}=\delta_{i j} \delta_{r s} 2 E_{i}(\mathbf{p}) \delta^{3}\left(\mathbf{p}-\mathbf{p}^{\prime}\right) ;
\end{aligned}
$$

all other anticommutation relations are null. The functions $u, v$ are defined as

$$
\begin{gathered}
u_{i}^{s}(x ; \mathbf{p})=u_{i}^{s}(\mathbf{p}) \frac{e^{-i p_{i} \cdot x}}{(2 \pi)^{3 / 2}}, \\
u_{i}^{s}(\mathbf{p})=\frac{m_{i}+E_{i} \gamma^{0}-\mathbf{p} \cdot \gamma}{\sqrt{E_{i}+m_{i}}} u_{0}^{s}, \\
v_{i}^{s}(x ; \mathbf{p})=v_{i}^{s}(\mathbf{p}) \frac{e^{i p_{i} \cdot x}}{(2 \pi)^{3 / 2}}, \\
v_{i}^{s}(\mathbf{p})=\frac{m_{i}-E_{i} \gamma^{0}+\mathbf{p} \cdot \gamma}{\sqrt{E_{i}+m_{i}}} v_{0}^{s},
\end{gathered}
$$

where $p_{i} \cdot x=E_{i}(\mathbf{p}) t-\mathbf{p} \cdot \mathbf{x}$ and they satisfy the properties

$$
\begin{gathered}
\bar{u}_{0}^{r} u_{0}^{s}=u_{0}^{r \dagger} u_{0}^{s}=-\bar{v}_{0}^{r} v_{0}^{s}=v_{0}^{r \dagger} v_{0}^{s}=\delta_{r s}, \\
v_{0}^{r \dagger} u_{0}^{s}=u_{0}^{r \dagger} v_{0}^{s}=0 \quad \forall r, s, \\
\sum_{s} u_{i}^{s}(\mathbf{p}) \bar{u}_{i}{ }^{s}(\mathbf{p})=\not p+m_{i}=2 E_{i}(\mathbf{p}) \Lambda_{i+}^{D}(\mathbf{p}) \gamma^{0}, \\
\sum_{s} v_{i}^{s}(\mathbf{p}) \bar{v}_{i}^{s}(\mathbf{p})=\not p-m_{i}=2 E_{i}(\mathbf{p}) \Lambda_{i-}^{D}(-\mathbf{p}) \gamma^{0} .
\end{gathered}
$$

The Feynman propagator for fermions is

$$
i S_{F}(x-y) \equiv\langle 0|T(\psi(x) \bar{\psi}(y))| 0\rangle
$$




$$
\begin{gathered}
=\int \frac{d^{4} p}{(2 \pi)^{4}} \frac{i}{\not p-m+i \epsilon} e^{-i p \cdot(x-y)} \\
=(i \not \supset+m) i \Delta_{F}(x-y ; m) .
\end{gathered}
$$

The function $S$ in Eq. (34) and its equivalent for the Sakata-Taketani Hamiltonian can be written as

$$
\begin{gathered}
i S(x ; m)=(i \not \supset+m) i \Delta(x ; m), \\
K^{\mathrm{ST}}(x ; m)=\left[i \partial_{t}-\frac{\nabla^{2}}{2 m}\left(\tau_{3}+i \tau_{2}\right)+m^{2}\right] i \Delta(x ; m), \\
i \Delta(x ; m)=\frac{1}{(2 \pi)^{3}} \int d^{4} p \delta\left(p^{2}-m^{2}\right) \boldsymbol{\epsilon}\left(p_{0}\right) e^{-i p \cdot x} \\
=\frac{1}{(2 \pi)^{3}} \int \frac{d \mathbf{p}}{2 E_{p}}\left[e^{-i p \cdot x}-e^{+i p \cdot x}\right] .
\end{gathered}
$$

The free neutrino eigenstates are defined as

$$
\begin{gathered}
\left|\nu_{i}(\mathbf{p}, s)\right\rangle \equiv \frac{a_{i}^{\dagger}(\mathbf{p}, s)}{\sqrt{2 E_{i}}}|0\rangle \\
=\int d \mathbf{x} \nu_{i}^{\dagger}(x)|0\rangle \frac{u_{i}(x ; \mathbf{p})}{\sqrt{2 E_{i}^{-}}}, \\
\left|\bar{\nu}_{i}(\mathbf{p}, s)\right\rangle \equiv \frac{b_{i}^{\dagger}(\mathbf{p}, s)}{\sqrt{2 E_{i}}}|0\rangle \\
=\int d \mathbf{x} \frac{v_{i}^{\dagger}(x ; \mathbf{p})}{\sqrt{2 E_{i}}} \nu_{i}(x)|0\rangle,
\end{gathered}
$$

whose normalization is $\left\langle\nu_{j}(\mathbf{p}, r) \mid \nu_{i}\left(\mathbf{p}^{\prime}, s\right)\right\rangle=\delta_{i j} \delta_{r s} \delta^{3}(\mathbf{p}-$ $\left.\mathbf{p}^{\prime}\right)$. The same normalization is valid for the antiparticle states. The states with finite momentum distributions are defined as

$$
\begin{aligned}
\left|\nu_{i}: g\right\rangle & =\sum_{s} \int d \mathbf{p} g^{s}(\mathbf{p})\left|\nu_{i}(\mathbf{p}, s)\right\rangle \\
= & \int d \mathbf{x} \nu_{i}^{\dagger}(x)|0\rangle \psi_{\nu_{i}}(x), \\
\psi_{\nu_{i}}(x) & \equiv \sum_{s} \int d \mathbf{p} g^{s}(\mathbf{p}) \frac{u_{i}^{s}(x ; \mathbf{p})}{\sqrt{2 E_{i}}}, \\
e^{-i H t}\left|\nu_{i}: g\right\rangle & =\int d \mathbf{x} \nu_{i}^{\dagger}(\mathbf{x}, 0)|0\rangle \psi_{\nu_{i}}(\mathbf{x}, t), \\
\left|\bar{\nu}_{i}: g\right\rangle & =\sum_{s} \int d \mathbf{p} g^{s *}(\mathbf{p})\left|\bar{\nu}_{i}(\mathbf{p}, s)\right\rangle \\
& =\int d \mathbf{x} \psi_{\bar{\nu}_{i}}^{\dagger}(x) \nu_{i}(x)|0\rangle,
\end{aligned}
$$

$$
\begin{array}{r}
\psi_{\bar{\nu}_{i}}(x) \equiv \sum_{s} \int d \mathbf{p} g^{s}(\mathbf{p}) \frac{v_{i}^{s}(x ; \mathbf{p})}{\sqrt{2 E_{i}}}, \\
e^{-i H t}\left|\bar{\nu}_{i}: g\right\rangle=\int d \mathbf{x} \psi_{\bar{\nu}_{i}}^{\dagger}(\mathbf{x}, t) \nu_{i}(\mathbf{x}, 0)|0\rangle .
\end{array}
$$

\section{APPENDIX B: DECOMPOSITION WITH RESPECT TO TWO BASES}

It is possible to decompose a given spinorial function $\psi(\mathbf{x})$ in terms of bases depending on different masses $m_{1}$ and $m_{2}$. Equating

$$
\begin{aligned}
\psi(\mathbf{x}) & =\int \frac{d \mathbf{p}}{2 E_{i}}\left[u_{i}^{s}(\mathbf{x} ; \mathbf{p}) g_{i}^{(+)}(\mathbf{p}, s)+v_{i}^{s}(\mathbf{x} ; \mathbf{p}) g_{i}^{(-)}(\mathbf{p}, s)\right], \\
i & =1,2,
\end{aligned}
$$

the expansion coefficients can be obtained:

$$
\begin{aligned}
& g_{i}^{(+)}(\mathbf{p}, s)=\int d \mathbf{x} u_{i}^{s \dagger}(\mathbf{x} ; \mathbf{p}) \psi(\mathbf{x}), \\
& g_{i}^{(-)}(\mathbf{p}, s)=\int d \mathbf{x} v_{i}^{s \dagger}(\mathbf{x} ; \mathbf{p}) \psi(\mathbf{x}) .
\end{aligned}
$$

From Eq. (B3) we see that imposing the conditions

$$
\begin{aligned}
& g_{1}^{(-)}(\mathbf{p}, s)=v_{1}^{s \dagger}(\mathbf{p}) \tilde{\psi}(-\mathbf{p})=0, \\
& g_{2}^{(-)}(\mathbf{p}, s)=v_{2}^{s \dagger}(\mathbf{p}) \tilde{\psi}(-\mathbf{p})=0,
\end{aligned}
$$

for all $\mathbf{p}$, leads to the equivalent conditions

$$
\left[\left(m_{1}+E_{2}\right)-\left(m_{2}+E_{2}\right)\right] v_{0}^{s \dagger} \tilde{\psi}(-\mathbf{p})=0, \quad s=1,2,
$$

$\left[\frac{1}{m_{1}+E_{2}}-\frac{1}{m_{2}+E_{2}}\right] \boldsymbol{v}_{0}^{s \dagger} \boldsymbol{\gamma} \cdot \mathbf{p} \tilde{\psi}(-\mathbf{p})=0, \quad s=1,2$,

where the properties of Eq. (A10) and $\gamma_{0} v_{0}^{s}=-v_{0}^{s}$ were used. In case $m_{1} \neq m_{2}$, we can use the decomposition $\tilde{\psi}(\mathbf{p})=\tilde{\psi}_{+}(\mathbf{p})+\tilde{\psi}_{-}(\mathbf{p}), \quad$ where $\quad \tilde{\psi}_{ \pm}(\mathbf{p})=(1 \pm$ $\left.\gamma_{0}\right) \tilde{\psi}(\mathbf{p}) / 2$, and obtain from Eqs. (B6) and (B7) the conditions

$$
\begin{gathered}
v_{0}^{s \dagger} \tilde{\psi}_{-}(-\mathbf{p})=0, \quad s=1,2, \\
u_{0}^{s \dagger} \sigma \cdot \mathbf{p} \tilde{\psi}_{+}(-\mathbf{p})=0, \quad s=1,2,
\end{gathered}
$$

where the properties $\boldsymbol{\gamma}=\gamma_{0} \gamma_{5} \boldsymbol{\sigma}$ and $u_{0}^{s}=\gamma_{5} \boldsymbol{v}_{0}^{s}$ were used in Eq. (B9). Equations (B8) and (B9) are only satisfied if $\tilde{\psi}_{+}(\mathbf{p})=\tilde{\psi}_{-}(\mathbf{p})=0$, since $\boldsymbol{\sigma} \cdot \mathbf{p}$ has only non-null eigenvalues and it commutes with $\gamma_{0}$. It is easier to reach this conclusion in the helicity basis $\left\{u_{0}^{( \pm)}, v_{0}^{( \pm)}\right\}$characterized by $\boldsymbol{\sigma} \cdot \mathbf{p} u_{0}^{( \pm)}= \pm|\mathbf{p}| u_{0}^{( \pm)}$, but the result is basis independent. 


\section{APPENDIX C: INTEGRALS}

Splitting the Feynman propagator into positive and negative frequency parts $i S_{F}(x)=i S^{(+)}(x)+i S^{(-)}(x)$, the following integrals give us

$$
\begin{aligned}
\int d t e^{i \omega t} i S^{(+)}(\mathbf{r}, t ; m)= & (-i) \frac{e^{i k_{\omega} r}}{4 \pi r}\left[\left(\omega \gamma_{0}-k_{\omega}(\hat{\mathbf{r}} \cdot \gamma)\right.\right. \\
& +m] \theta(\omega-m) \\
\int d t e^{i \omega t} i S^{(-)}(\mathbf{r}, t ; m)= & (-i) \frac{e^{i k_{\omega} r}}{4 \pi r}\left[\left(\omega \gamma_{0}-k_{\omega}(\hat{\mathbf{r}} \cdot \gamma)\right.\right. \\
& +m] \theta(-\omega-m),
\end{aligned}
$$

where $k_{\omega}=\sqrt{\omega^{2}-m^{2}}, \mathbf{r}=r \hat{\mathbf{r}}$, the conditions $m r, k_{\omega} r \gg$ 1 were assumed, and terms behaving as $1 / r^{2}$ were neglected.

To illustrate the calculations, Eq. (C1) is obtained by

$$
\begin{aligned}
\int d t e^{i \omega t} i S^{(+)}(\mathbf{r}, t ; m)= & \frac{1}{(2 \pi)^{3}} \int \frac{d \mathbf{p}}{2 E(\mathbf{p})} \\
& \times \frac{i\left(E(\mathbf{p}) \gamma_{0}-\mathbf{p} \cdot \gamma+m\right)}{\omega-E(\mathbf{p})+i \epsilon} e^{i \mathbf{p} \cdot \mathbf{r}}
\end{aligned}
$$

$$
\begin{aligned}
= & \frac{1}{(2 \pi)^{2}} \frac{-i}{2 r} \int_{-\infty}^{\infty} d p \frac{p}{E(p)}\left\{\sin p r \frac{E(p) \gamma_{0}+m}{E(p)-\omega-i \epsilon}\right. \\
& \left.+\left[\cos p r-\frac{\sin p r}{p r}\right] \frac{i p(\hat{\mathbf{r}} \cdot \gamma)}{E(p)-\omega-i \epsilon}\right\} .
\end{aligned}
$$

In Eq. (C3) the following identity is used:

$$
\int_{0}^{\infty} d t e^{ \pm i E t}=\frac{ \pm i}{E \pm i \epsilon}
$$

To get to the result of Eq. (C1) it is necessary to split the functions $\sin p r$ and $\cos p r$ in Eq. (C4) into exponentials and, for the $e^{i p r}$ part, integrate along a closed path formed by a half semicircle in the upper-half complex plane going round the branching line $\left[i m, i \infty\right.$ ) (for the $e^{-i p r}$ part take the path reflected by the line defined by $\operatorname{Rez}=0$ ). The contribution from the paths beside the branching line yields a function which decreases more rapidly than $e^{-m r}$ and it is negligible for $m r \gg 1$. The contributions for $-m<\omega<$ $m$ give a function with dependence $e^{-\left|k_{\omega}\right| r}$ which is also negligible for large separations $r$.
[1] R. J. Davis, D. S. Harmer, and K. C. Hoffman, Phys. Rev. Lett. 20, 1205 (1968); S. Fukuda et al. (SuperKamiokande Collaboration), Phys. Rev. Lett. 86, 5656 (2001); Q. R. Ahmad et al. (SNO Collaboration), Phys. Rev. Lett. 87, 071301 (2001); 89, 011301 (2002); K. Eguchi et al. (KamLAND Collaboration), Phys. Rev. Lett. 90, 021802 (2003); M.H. Ahn et al. (K2K Collaboration), Phys. Rev. Lett. 90, 041801 (2003).

[2] A. Y. Smirnov, Int. J. Mod. Phys. A 19, 1180 (2004).

[3] M. Blasone and G. Vitiello, Ann. Phys. (N.Y.) 244, 283 (1995); 249, 363(E) (1996).

[4] M. Blasone and J.S. Palmer, Phys. Rev. D 69, 057301 (2004).

[5] B. Pontecorvo, Zh. Eksp. Teor. Fiz. 33, 549 (1957) [Sov. Phys. JETP 6, 429 (1957)]; Z. Maki, M. Nakagawa, and S. Sakata, Prog. Theor. Phys. 28, 870 (1962); S. M. Bilenky and B. Pontecorvo, Phys. Rep. 41, 225 (1978).

[6] K. Hagiwara et al. (Particle Data Group), Phys. Rev. D 66, 010001 (2002).

[7] B. Kayser, Phys. Rev. D 24, 110 (1981).

[8] C. Giunti, C. W. Kim, and U. W. Lee, Phys. Rev. D 44, 3635 (1991).

[9] S. De Leo, C. C. Nishi, and P. P. Rotelli, Int. J. Mod. Phys. A 19, 677 (2004).

[10] M. Zralek, Acta Phys. Pol. B 29, 3925 (1998).

[11] K. Kiers and N. Weiss, Phys. Rev. D 57, 3091 (1998).

[12] C. Giunti and C. W. Kim, Phys. Rev. D 58, 017301 (1998).

[13] J. Rich, Phys. Rev. D 48, 4318 (1993).
[14] J. H. Field, Eur. Phys. J. C 30, 305 (2003).

[15] C. Giunti, C. W. Kim, J. A. Lee, and U. W. Lee, Phys. Rev. D 48, 4310 (1993).

[16] J. E. Campagne, Phys. Lett. B 400, 135 (1997).

[17] See, for a review, M. Beuthe, Phys. Rep. 375, 105 (2003).

[18] I. Y. Kobzarev, B. V. Martemyanov, L. B. Okun, and M. G. Shchepkin, Yad. Fiz. 35, 1210 (1982) [Sov. J. Nucl. Phys. 35, 708 (1982)].

[19] W. Grimus and P. Stockinger, Phys. Rev. D 54, 3414 (1996); W. Grimus, P. Stockinger, and S. Mohanty, Phys. Rev. D 59, 013011 (1999).

[20] A. D. Dolgov, L. B. Okun, M. V. Rotaev, and M.G. Schepkin, hep-ph/0407189; A.D. Dolgov, O. V. Lychkovskiy, A. A. Mamonov, L. B. Okun, M. V. Rotaev, and M. G. Schepkin, Nucl. Phys. B729, 79 (2005).

[21] C. Y. Cardall, Phys. Rev. D 61, 073006 (2000).

[22] A.E. Bernardini and S. De Leo, Eur. Phys. J. C 37, 471 (2004).

[23] A. E. Bernardini and S. De Leo, Phys. Rev. D 71, 076008 (2005).

[24] H. Feshbach and F. Villars, Rev. Mod. Phys. 30, 24 (1958); S. Sakata and M. Taketani, Proc. Phys. Math. Soc. Jpn. 22, 757 (1940).

[25] M. Dvornikov, Phys. Lett. B 610, 262 (2005).

[26] R. N. Mohapatra and P.B. Pal, Massive Neutrinos in Physics and Astrophysics, Lecture Notes in Physics (World Scientific, Singapore, 1991), Vol. 41, p. 1.

[27] S. De Leo and P. Rotelli, Int. J. Theor. Phys. 37, 2193 
(1998).

[28] C. Itzykson and J.B. Zuber, Quantum Field Theory (McGraw-Hill, New York, 1980), p. 60.

[29] L. L. Foldy and S. A. Wouthuysen, Phys. Rev. 78, 29 (1950).

[30] B. Thaller, The Dirac Equation (Springer-Verlag, Berlin, 1992).

[31] The charge here is a global U(1) charge which is conserved for $n=1,2$ and does not mean electric charge. Obviously the flavor charges $\alpha=e, \mu$, introduced by mixing, depend on time and quantifies the oscillation, as in Eq. (31).

[32] P. Roman, Introduction to Quantum Field Theory (Wiley, New York, 1969), pp. 27-48.

[33] C. Giunti, J. High Energy Phys. 11 (2002) 017.

[34] M. Blasone and G. Vitiello, Phys. Rev. D 60, 111302(R) (1999); M. Blasone, P. Jizba, and G. Vitiello, hep-ph/ 0308009.

[35] S. Weinberg, Quantum Theory of Fields (Cambridge University Press, Cambridge, England, 1995), Vol. I, Chap. 4. 